\title{
High-value B2B interactions, non-repudiation and Web services
}

\author{
Nick Cook \\ School of Computing Science \\ Newcastle University \\ Newcastle, NE1 7RU, UK \\ tel: +441912464909 \\ fax: +44 1912228232 \\ email: nick.cook@ncl.ac.uk
}

Paul Robinson

Arjuna Technologies

Nanotechnology Centre

Newcastle, NE1 7RU, UK

tel: +441912430676

fax: +441912430677

email: paul.robinson@arjuna.com

Santosh K. Shrivastava

School of Computing Science

Newcastle University

Newcastle, NE1 7RU, UK

tel: +44 1912228038

fax: +44 1912228232

email: santosh.shrivastava@ncl.ac.uk 


\title{
High-value B2B interactions, non-repudiation and Web services
}

\begin{abstract}
This chapter provides an overview of the problem of making high-value business-to-business (B2B) interactions non-repudiable, where non-repudiation is the property that no party to an interaction can subsequently deny their involvement in the interaction. Existing approaches are discussed in the context of fundamental work on fairness and non-repudiation. The existing work suffers from a lack of flexibility both in terms of the mechanisms that can be deployed to achieve non-repudiation and of the interactions to which non-repudiation can be applied. The authors contend that it is necessary to be able to render arbitrary Web service interactions non-repudiable and to optionally invoke application-level validation of business messages at run-time. The chapter presents the design and implementation of a novel Web services-based middleware that addresses these requirements. The middleware leverages existing Web service standards. It is sufficiently flexible to adapt to different regulatory regimes and to provide security guarantees that are appropriate to different business contexts.
\end{abstract}

Keywords: B2B E-Commerce; Middleware; Distributed Systems; Security; Internet Security; Non-repudiation; XML

\section{INTRODUCTION}

It is increasingly common to structure business-to-business (B2B) functions in terms of welldefined business message exchanges between loosely coupled services. This has led to the development of open standards for business conversations. For example, the RosettaNet Partner Interface Process standards (RosettaNet, 2005) define observable B2B interactions in terms of the XML messages that business partners should exchange in order to perform functions such as product line querying and order processing. Business partners use such standards as the basis for agreement on the syntax, semantics and sequencing of messages; and on the $\mathrm{B} 2 \mathrm{~B}$ processes that they should execute. A problem that then arises is how to ensure that an executing interaction complies with these business agreements. Monitoring for, and enforcement of, compliance implies that interacting parties must be held to account for their actions. That is, it should not be possible to deny participation in a B2B interaction. In this context, non-repudiation services provide protection against false denial of involvement in communication. A related concern is that honest parties should not suffer disadvantage due to the misbehaviour, or non-cooperation, of others.

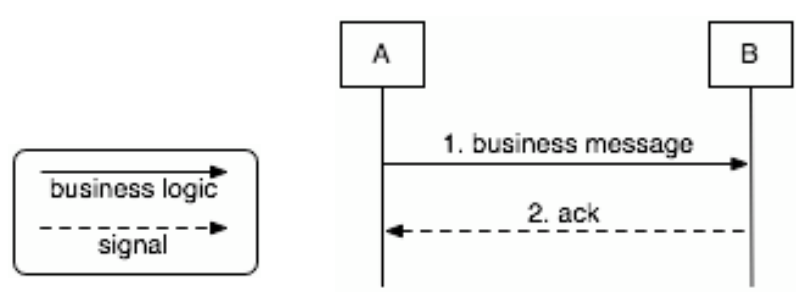

(a) Business message with acknowledgement

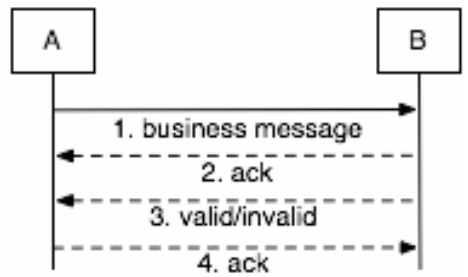

(b) Business message validation and acknowledgement

Figure 1: Typical B2B interactions

A typical business message exchange between organisations A and B is shown in Figure 1a. There are two types of message flow: solid lines show the flow of business logic messages 
and dashed lines the flow of signal messages for acknowledgement of receipt. Party A sends a business message to party B and B provides an acknowledgement of receipt (ack) in return. Figure $1 \mathrm{~b}$ shows an extended interaction where B both acknowledges A's business message and, in step 3, asserts its validity, or otherwise, with respect to agreements governing the interaction. Message validity may relate to some business specific constraints on the content of messages and/or may be with respect to some contract that governs a cross-organisational business process composed of a number of message exchanges. Essentially, if B signals the validity of a message they confirm that the message is acceptable for continued processing in the business context. As shown, $\mathrm{A}$ is expected to provide an ack for the validation message in step 4. Standards such as RosettaNet define processes in terms of message exchanges that can be mapped to one of the types shown in Figure 1. Now, consider the scenario in which A and $\mathrm{B}$ do not unguardedly trust each other and either party may misbehave in some way. For example, B may receive the business message from A but decline to provide the ack. This is the selective receipt problem and places $\mathrm{A}$ at a disadvantage. Also, unless there is irrefutable evidence of the origin of A's message, $\mathrm{A}$ is able to subsequently deny their involvement in the exchange. Similarly, irrefutable evidence of the origin of ack is required to subsequently demonstrate B's involvement. The same concerns arise in relation to the exchange of B's validation message for A's ack. To address these problems and, thereby, safeguard the interests of both A and B, the simple business message exchange should be made both fair and non-repudiable. Informally, fairness is the property that well-behaved parties are not disadvantaged by the behaviour of misbehaving parties. Non-repudiation is the property that an action or event cannot subsequently be denied. The RosettaNet standard specifies whether non-repudiation is mandatory or optional for a given message exchange. It does not specify the mechanism to achieve non-repudiation or additional properties such as fairness.

Businesses are adopting Web services as a platform for high-value B2B interactions of the above kind. WS-Security specifies how to apply message-level security. The XML Key Management Specification (XKMS) brings PKI functionality to Web services. The Digital Signature Service (DSS) specifies services for signature verification and secure time-stamps. Implementations of these Web service specifications, and others such as WSReliableMessaging and WS-Addressing, represent an essential supporting infrastructure. However, this infrastructure is not sufficient to address the regulatory requirements of highvalue B2B interactions. We need additional mechanisms to ensure compliance with business agreements. For example, existing standards cannot guarantee that outcomes are fair to honest participants. Such guarantees are necessary to reduce, if not eliminate, the need to resort to costly dispute resolution. This chapter explores the regulatory mechanisms that are necessary to achieve non-repudiation and fairness in Web service interactions. It also shows how these mechanisms can be used as building blocks for compliance with business agreements.

The chapter provides an overview of existing solutions to the problem of regulation of $\mathrm{B} 2 \mathrm{~B}$ interactions. The shortcomings of such solutions are discussed in the context of fundamental work on fairness and non-repudiation. We motivate our work with a real-world scenario that can be reduced to interactions of the type shown in Figure 1. The scenario demonstrates the need for different approaches, with different security characteristics, to making such interactions non-repudiable. We describe protocols that are representative of these different approaches. We then present our novel middleware for the regulation of interactions. The middleware leverages Web service standards. It is sufficiently flexible to execute different protocols to suit different regulatory regimes and to provide security guarantees that are appropriate to the given business context. The chapter concludes with suggestions for some further work. 


\section{BACKGROUND}

This section introduces background to our work, concentrating on examples of existing middleware services for non-repudiable interaction. We identify shortcomings of existing work that are addressed by the Web services solution presented in the remainder of the chapter. First, we briefly discuss fundamental work on non-repudiation and fairness that is the basis for the protocols for non-repudiable B2B interactions.

\section{Non-repudiation and fairness}

Asokan (Asokan, 1998) provides one of the most widely accepted informal definitions of fairness: that a system is fair if it does not discriminate against correctly behaving parties. More recently, Markowitch et al (Markowitch, Gollmann, \& Kremer, 2002) provide an overview of the evolution of the notion of fairness in exchange and the various definitions that have been proposed. To clarify the position, they propose a definition of three mandatory properties that must be satisfied for an exchange protocol to be considered secure: viability, timeliness and fairness. Viability means that it is possible for an execution of the protocol to result in successful exchange, irrespective of the quality of communication channels. Timeliness is the property that, with the quality of the communication channel fixed, there is always a point in the protocol that can be reached in a finite amount of time when parties can stop the protocol without compromising fairness. The definition of the fairness property is that, with communication channel quality fixed, at the end of the exchange protocol run, either all participants obtain their expected items or they receive none of the information to be exchanged with respect to missing items. They define non-repudiability as the optional property of a secure exchange that it is impossible for any participant to subsequently deny having participated in a part or the whole of the communication. For non-repudiation of delivery of a single business message, the sender provides the message and non-repudiation of origin evidence in exchange for non-repudiation of receipt evidence from the recipient. The fairness guarantee for the sender is that the recipient must provide a valid receipt in order to obtain the message. The corresponding fairness guarantee for the recipient is that the sender must provide valid evidence of origin in order to obtain the receipt.

There is an extensive literature on the problem of fair exchange and its solution. Kremer et al (Kremer, Markowitch, \& Zhou, 2002) provide a detailed survey of fair non-repudiation protocols. Wang (Wang, 2005) includes an updated comparison of deterministic protocols. The role of a Trusted Third Party (TTP) as guarantor of fairness is central to much of the work on fair exchange. Even and Yacobi (Even \& Yacobi, 1980) provide the fundamental insight that deterministic fair exchange is impossible without a guarantor TTP. This impossibility result is supported by arguments that relate the problem to the Fischer-LynchPaterson (FLP) impossibility result for distributed consensus (Ezhilchelvan \& Shrivastava, 2005; Fischer, Lynch, \& Paterson, 1985; Pagnia \& Gärtner, 1999). Deterministic protocols can be characterised by the level of active involvement of the TTP in an exchange in the normal case. An in-line TTP is involved in every message exchange, relaying messages between the other participants. An on-line TTP is typically only involved in protocol set-up. An off-line TTP is not involved in the normal case and is only called up on to recover fairness for honest participants should normal execution fail. This is the optimistic approach to fair exchange. In-line and on-line TTPs also recover fairness for honest parties. Recovery of fairness means that the TTP (whether in-line, on-line or off-line) guarantees that an exchange can terminate without disadvantage to honest parties. The TTP ensures either that honest parties can obtain the items they expect from an exchange or that no party gains any 
useful additional information from the exchange. This recovery is normally achieved through the execution of sub-protocols between protocol participants and the TTP.

The impossibility result and the desire to eliminate the potential bottleneck of a guarantor TTP have led protocol developers to concentrate on the reduction of the involvement of the TTP. This is the main motivation for the optimistic approach to deterministic fair exchange. Other developers have adopted a probabilistic approach that eliminates the need for a guarantor TTP altogether ((Markowitch \& Roggeman, 1999) presents an early example). The trade-off for elimination of the guarantor TTP is that there is a non-zero probability of a loss of fairness. Probabilistic protocols are also more communication-intensive than the protocols that offer a deterministic fairness guarantee. Implementation of probabilistic protocols is beyond the scope of this chapter and we will not discuss them further.

Non-repudiation relies on maintaining the integrity of signed evidence beyond the execution of the given exchange. Since it is possible for a key to become compromised before its expiry, most signature schemes allow the owner of a key to revoke the key. Keys are usually revoked by sending a request to revoke the related certificate to some Certificate Authority. Parties who rely on the validity of a signature also refer to the Certificate Authority for key revocation information. In most commonly used signature schemes, such as the X509 PKI (Housley, Ford, Polk, \& Solo, 1999), there is no obligation on the owner of a public key to inform relying parties that they have revoked the corresponding private key. The ability to revoke a private key without reference to relying parties can compromise the long-term integrity of evidence signed with the key. A signing party can use their private key to generate a signature and a relying party can verify the signature upon receipt. However, the signing party can subsequently revoke the key. Without evidence of the time of use of the key, a relying party cannot prove that the signature was generated before key revocation and the signing party can repudiate the evidence. For this reason, if a signing key is revocable, a trusted time-stamp must be applied to signed evidence to demonstrate that the key was not compromised at time of use. Given A's signature $s$ on some data $x\left(s=\operatorname{sig}_{A}(x)\right)$, a TTP timestamping authority, TSA, can generate the time-stamp: $\left\{T_{g}, \operatorname{sig}_{T S A}\left(s, T_{g}\right)\right\}$ as proof of the generation (or existence) of $s$ at time $T_{g}$ (Zhou \& Gollmann, 1997). Assuming A's key was not revoked at time $T_{g}$, the trusted time-stamp prevents A from subsequently denying the validity of $s$. A TSA is an on-line TTP. A TTP that provides time-stamping services is a candidate for the provision of other third party services (including that of guarantor TTP for fair exchange protocols). For brevity, we do not show trusted time-stamps in the protocol descriptions. However, they are applied to signatures by our middleware framework during protocol execution.

\section{The FIDES research project fair exchange service}

The FIDES research project (Nenadic, Zhang, \& Barton, 2004) provides services, including TTP services, and an associated application for fair exchange of business items (documents). The project addresses two problems: (i) the development of a family of deterministic fair exchange protocols, and (ii) the design and implementation of a system for execution of the protocols. Each enterprise hosts a FIDES server and a set of one or more FIDES clients. A TTP service is available for dispute resolution. Within an enterprise, users interact with the system through GUI-based clients that provide secure access to the services hosted by their local FIDES server. The FIDES server at one enterprise executes fair exchange protocols with a peer server at another enterprise. FIDES servers also execute recovery protocols with the TTP service. Items for exchange are stored in a secure database. FIDES services are implemented on a J2EE application server. Java Messaging Service (JMS) is used for both client-to-server and server-to-server communication. Application clients submit documents to 
their local FIDES system for fair exchange with partners who also have a FIDES client for verifying and receipting the documents they receive. In effect, FIDES offers a standalone service for fair exchange.

A drawback of the approach is that the only interaction with the exchange protocol service is through FIDES application clients. There is no published API for client-side interaction with a FIDES server or for the server-to-server execution of protocols. Users are therefore restricted to an application-specific mechanism for the exchange of items. Other than user agreement to involvement in an exchange, there is no support for run-time validation of the interaction with respect to contract. Any contractual constraints that should be imposed must be dealt with during negotiation of the items to be exchanged, and of the parameters of the exchange. Nevertheless, as far as we are aware, the FIDES project and our work presented in this chapter represent the only service-based implementations of fair exchange.

\section{Typical commercial approach}

BEA's WebLogic Trading Partner Integration Engine (TPIE) (BEA, 2005) is typical of commercial approaches to the regulation of B2B interactions. As shown in Figure 2 the Trading Partner Integration Engine (TPIE) is integrated with the application server. Nonrepudiation is provided as a component of the TPIE.

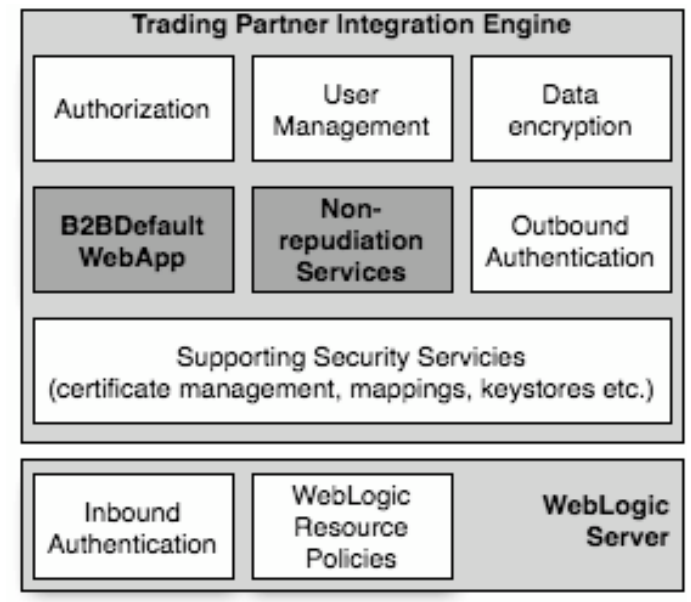

Figure 2: BEA WebLogic Trading Partner Integration Engine

In the BEA system, trading partners agree protocol bindings for their business conversations. Currently they provide bindings for the ebXML (Grangard, Eisenberg, \& Nickull, 2001) and RosettaNet PIP standards. The protocol binding to use at run-time is then configured in the B2BDefaultWebApp component of each partner's TPIE. The trading partners have the option to apply signatures to the messages of the given business protocol. The non-repudiation service handles the application and verification of signatures at run-time. The service also provides secure audit and time-stamping services. Interfaces to these support services have been defined to allow the plug-in of third-party providers. The BEA system is interesting because they provide non-repudiation of XML-based business message exchanges that are validated with respect to the business protocol binding in use. This is a form of regulated $\mathrm{B} 2 \mathrm{~B}$ interaction. The non-repudiation is voluntary. There is no support for fair exchange and there is no mechanism to introduce protocols to provide fair exchange. In effect, nonrepudiation of receipt is only available when acknowledgements are specified as part of the run-time business protocol binding. Similarly, validation with respect to contract is only available for the supported business protocol bindings. It is not possible to apply systematic validation with respect to other types of contract or agreement, or to make such validated 
interactions non-repudiable. In fact, it is not clear whether it is possible to verify that some agreed composition of PIP exchanges is adhered to correctly.

\section{Summary}

In this section, we provided an overview of related background work. The main drawback of these approaches is their inflexibility. FIDES is the only other work we are aware of that takes account of fundamental work on fair exchange and non-repudiation. They have taken what is in effect a human user centric, proprietary approach. Inter-organisational communication is not based on any Web service or other standard for the exchange of business messages. They provide a closed system that is not amenable to interaction with or between Web services.

The state of the art in industry is to provide voluntary non-repudiation. At the basic level this involves the signing of outgoing messages and the verification of incoming messages equivalent to voluntary non-repudiation of origin of messages. Aside from the BEA offering, there are commercial solutions that will provide these operations as part of an XML firewall (see: (Verisign, 2004) and (DataPower, 2004)). These solutions do not provide the systematic exchange of evidence to achieve non-repudiation of receipt or to guarantee fairness.

However, protocol execution could be built on top of such solutions.

BEA and others in the application server market do provide implementations of standards such as RosettaNet PIP and ebXML that provide a form of validation with respect to business agreement. As noted, voluntary non-repudiation of receipt is also supported when dictated by the standard being used. There is no provision for general support for validation with respect to contract or the systematic support for non-repudiable exchange described in this chapter. The identified drawbacks have led to our systematic and flexible approach to non-repudiable message exchange and the validation of messages with respect to business agreements. We contend that it is necessary to be able to render arbitrary Web service interactions nonrepudiable and to optionally invoke application-level validation at run-time. This validation must be correctly correlated with the non-repudiation evidence and, to provide systematic support, the underlying mechanism should be agnostic about the validation process. Further, as will be apparent from the discussion in following section, different underlying mechanisms may be required even by the same interacting entities in different contexts. The remainder of this chapter presents a middleware solution that addresses these requirements

The ability to invoke arbitrary application-level validation of messages at run-time is a key feature of our approach. This is essentially an extensibility mechanism that allows the middleware to become an enforcement engine not only for business standards such as RosettaNet but also for other forms of electronic contract. We do not specify how contracts are formulated, verified or encoded. This is the subject of related but separate research on contract-mediated interaction, examples include: (Minsky \& Ungureanu, 2000), (Milosevic, Gibson, Linnington, Cole, \& Kulkarni, 2004) and (Molina-Jimenez, Shrivastava, \& Warne, 2005)

\section{PROTOCOLS FOR NON-REPUDIABLE AND VALIDATED BUSINESS MESSAGE DELIVERY}

We now present three approaches to achieving the non-repudiation of interactions of the type described in the introduction to the chapter. The first approach uses voluntary nonrepudiation typical of current commercial solutions. The second approach uses an in-line TTP to guarantee the fairness of exchanges. This is a service-oriented approach where the 
responsibilities of the TTP can be adjusted to suit the capabilities of the exchange endpoints. The third approach involves the direct (optimistic) exchange of items between participants and the involvement of an off-line TTP to guarantee fairness. For each approach, we present protocols for the non-repudiable exchange of a business message for its receipt (see Figure 1a) and then for non-repudiation of a validated business message exchange (see Figure 1b). The protocol descriptions are not specific to Web services. We address their execution in our Web services middleware framework, including support for application-level validation of business messages, after discussing the protocols.

First, to provide a concrete context for the subsequent discussion, we present an example of B2B collaboration in chemical manufacturing. The example scenario demonstrates the need for a flexible solution to non-repudiation with the ability to execute different protocols to meet the requirements of different business relationships and application contexts. From this scenario, we derive a flexible interceptor-based model for protocol-based interactions and summarise the non-repudiation requirements. Protocol notation and assumptions then precede the protocol descriptions.

The protocols presented in this section are based on existing work published by (Coffey \& Saidha, 1996) and (Wang, 2005). The various extensions and modifications to the published protocols are based on work published in (Cook, Robinson, \& Shrivastava, 2006). It should be noted that, for brevity, the protocol descriptions in this section do not include the specification of trusted time-stamps. It is assumed that if revocable keys are used to generate signed evidence then time-stamps of the kind discussed previously will be included in protocol messages. An analysis of the costs of time-stamping is provided in the summary to this section.

\section{An example application scenario}

Increasingly, chemical companies are seeking to form alliances, or virtual organisations (VOs), that can rapidly prototype new chemical processes prior to full-scale manufacture of products. These alliances will typically include a lead company that sub-contracts work to consultants for process simulation and to equipment manufacturers to design prototype plants. The example presented here is derived from the study of a collaborative B2B process to determine the feasibility of developing a new chemical manufacturing facility (see the Gold project (Periorellis et al., 2006)).

Figure 3 represents a small part of the overall B2B process. In any scenario of this kind, we can distinguish between two types of participant: business partners and TTPs. The consultancy and the manufacturer, represented by white rectangles, are business partners who wish to engage in a mutually advantageous collaboration. To support the process they may agree to use TTP services. The Trusted Service Provider (TSP), represented by a grey rectangle, is a TTP. By definition, TTPs always fulfil the commitments that they make and will cooperate with other correctly behaving parties. In contrast, there is a risk that a business partner may cease cooperation or may be unable or unwilling to fulfil their commitments to the collaboration. 


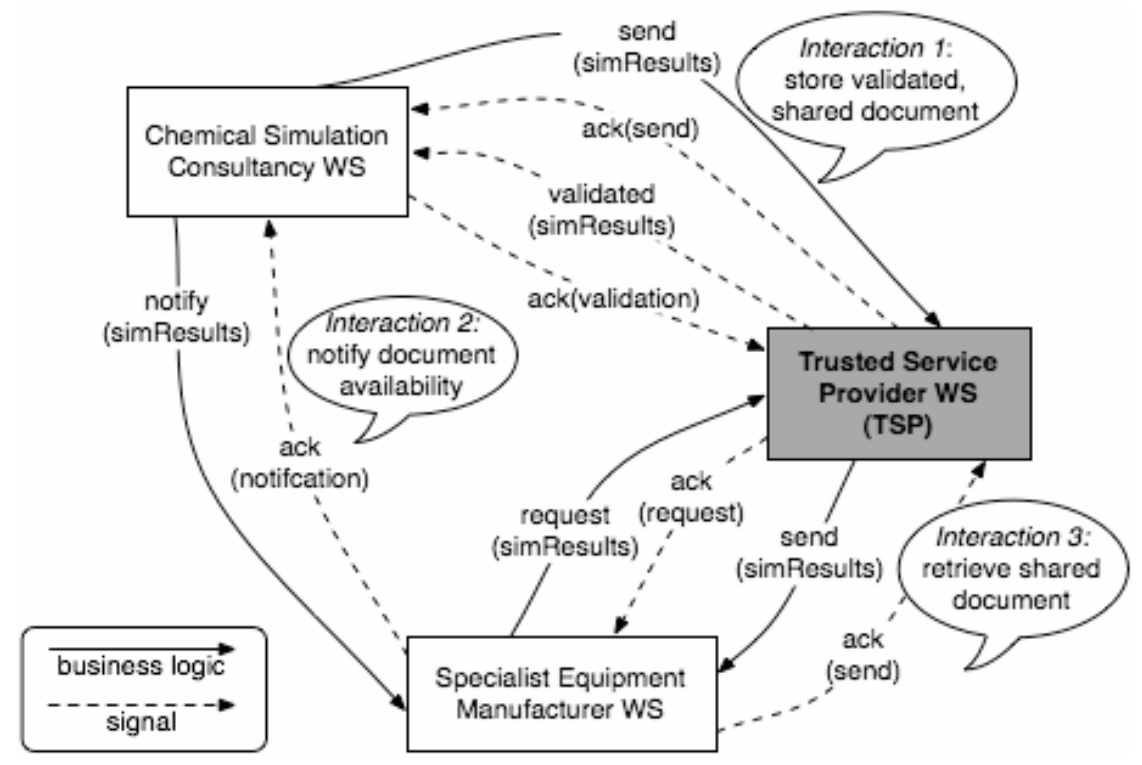

Figure 3: Chemical development WS interaction

All interactions in Figure 3 are between or with Web services that are hosted either by the business partners or by the TSP that supports the operation of the VO. The lead company (not shown) requires that all project documents (task outputs) must be placed in a shared repository hosted by the TSP that is responsible for managing access to the documents. The sub-process essentially illustrates the end of one task by a consultancy company and the resulting initiation of a subsequent task at a specialist equipment manufacturer. The consultancy company conducts simulation experiments. The output of these experiments is a simulation results report (simResults) that the consultancy stores with the TSP (interaction 1 in Figure 3). Storage of the document requires acknowledgement of receipt by the TSP and validation that the document complies with a structure specified by the lead company. The specialist equipment manufacturer must analyse the simResults to determine requirements for construction of a pilot plant for further experimental evaluation of the proposed new chemical manufacturing process. Interaction 2 in Figure 3 shows the consultancy company triggering this task by notifying the manufacturer of the availability of the validated simResults document. This task initiation must be acknowledged. Interaction 3 shows the manufacturer starting their task by retrieving the document from the TSP. The request for the document and its provision by the TSP must be acknowledged. As shown, each interaction entails a B2B message exchange of one of the types shown in Figure 1. Interaction 3, is a combination of two of the B2B message exchanges shown in Figure 1a.

The lead company requires that there is an audit trail of the critical interactions between organisations. Critical actions include the storage and retrieval of task outputs and the initiation of tasks. Furthermore, in order to irrefutably bind parties to the actions and commitments that are signified by the interactions, the audit trail must be non-repudiable. For example, the manufacturer's ack in Interaction 2 represents a commitment to start the task triggered by the availability of simResults. If the ack is non-repudiable then the manufacturer cannot deny receipt of the notification to signal the start of their involvement in the process.

In the preceding B2B process, the consultant and the manufacturer may not fulfil their commitments and disputes may arise. For example, there may be a dispute over whether all conditions were met for the initiation of analysis of the simResults by the manufacturer. To subsequently prove that they initiated the task correctly, the consultant must be able to show that they sent a valid simResults document to the TSP and that they notified the manufacturer of its availability. Assuming the consultant can provide this evidence, the manufacturer must be able to show that they started the task by retrieving the simResults document from the 
TSP. The generation and collection of non-repudiation evidence therefore serves both the interests of the lead company and of well-behaved participants in the process. However, the generation and collection of evidence involves the cooperation of both the TTP and the business partners. Each interaction in Figure 3 is composed from the exchange of a message for an acknowledgement of its receipt. We can group these message exchanges into three classes, characterised by the relationship between the parties: (i) business partner sender to TTP recipient (see phase 1 of Interaction 1 or 3); (ii) TTP sender to business partner recipient (see phase 2 of Interactions 1 or 3 ); and (iii) business partner sender to business partner recipient (see Interaction 2). Given the selective receipt problem, each class of message exchange requires a different underlying mechanism, or protocol, to achieve both nonrepudiation of origin of the sender's message (NRO) and non-repudiation of its receipt (NRR). For example, the consultant should be capable of using three different underlying mechanisms since it is involved in each of the three classes of message exchange. A fourth class of exchange that the TSP may engage in is TTP sender to TTP recipient. Therefore, in general, for any complex B2B process that involves two-way message exchange between different organisations, each organisation may need to engage in up to three of the different classes of message exchange.

\begin{tabular}{|l|l|l|}
\hline Sender & Recipient & Protocol type \\
\hline TTP & TTP & voluntary exchange \\
\hline business partner & TTP & voluntary exchange \\
\hline TTP & business partner & receipt-first exchange \\
\hline business partner & business partner & fair exchange \\
\hline
\end{tabular}

Table 1: Exchange classification

Table 1 shows the different classes of message exchange and the type of underlying exchange protocol that is required to provide both NRO and NRR in each case. We will provide examples of each protocol type, starting with voluntary exchange and then fair exchange. Finally, we will consider receipt-first exchange where a TTP obtains a receipt for an item before releasing the item. We will show that receipt-first exchange is a simplification of fair exchange. Of the protocol types, fair exchange offers the strongest guarantees. However, fair exchange can also be the most costly in terms of communications, computation and infrastructure. It therefore makes sense for the sender of a message to be able to adopt an exchange mechanism that is appropriate to their relationship with the intended recipient. Further, different capabilities may lead to the need to use different protocols even within a protocol type. As an example, we will show how to adapt a fair exchange protocol to different endpoint capabilities. That is, even in the context of a single B2B process, organisations may need to be flexible about the mechanisms that they use to achieve nonrepudiation. We now describe a model for interaction that can address this need for the flexibility of protocol-based non-repudiable interaction.

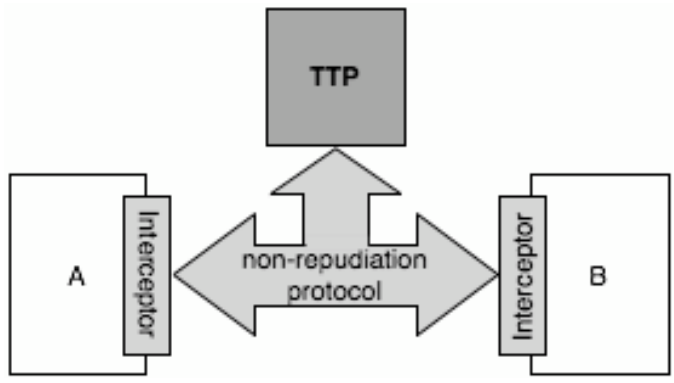

(b) Mediated non-repudiable interaction

(a) Business interaction

Figure 4: Interceptor-mediated non-repudiable interaction 
We are interested in business interactions that consist of one or more exchanges of messages for their acknowledgement. Figure 4a presents an abstract view of such interactions between two endpoint participants (A and B) in some B2B process. Our requirements are:

1. To render message exchange between $A$ and $B$ non-repudiable. That is, the sender of a business message should provide non-repudiation of origin (NRO) of the message and its recipient should provide non-repudiation of receipt (NRR).

2. To allow the use of different protocols to exchange non-repudiation evidence that are appropriate to different application and security contexts.

3. To support business message validation with respect to application-level agreements. Validation processes must be independent of non-repudiation mechanism yet validation decisions should be made non-repudiable. That is, we require nonrepudiation of origin of validation decisions (NROV) and non-repudiation of receipt of validation decisions (NRRV).

4. To provide the same high-level abstraction of business interaction between A and B shown in Figure 4a regardless of the underlying mechanisms used to achieve the exchange of non-repudiation evidence. That is, the application processes at either A or B should not be modified to meet the other requirements.

For delivery of a single business message, the precise application-level semantics are that the business message is only processed by the recipient application if the exchange of all related non-repudiation evidence succeeds. When validation is incorporated in the exchange, the business message is only processed if the message is deemed valid with respect to some application-specific validation process. That is, if there is a failure in exchange or validation, from the application viewpoint it is as if delivery of the related business message failed. From the application viewpoint, for the business and signal messages shown in Figure 1 and 3, either all the messages are valid and are delivered successfully, or the interaction fails.

To address these requirements we adopt an interceptor-based approach. As shown in Figure $4 \mathrm{~b}$, interceptors at A and B mediate their involvement in a non-repudiation exchange protocol that may also involve a TTP (whether in-line, on-line or off-line). The interceptor has two main functions: (i) to protect the interests of the party on whose behalf it acts by executing appropriate protocols, invoking validation processes and accessing appropriate services, including TTP services; and (ii) to hide the detail of the underlying mechanisms. The framework we present in this chapter is a Web services realisation of this model. The following protocol descriptions are an indication of the range of mechanisms that can be deployed in the framework. As we shall see, for business partner to business partner interactions, it is a TTP responsibility to deliver fairness guarantees. The introduction of interceptors allows us to hide such protocol details. For simplicity, in the following protocol descriptions we make no distinction between $\mathrm{A}$ and the interceptor that acts on their behalf, or between $\mathrm{B}$ and their interceptor.

\section{Protocol assumptions and notation}

We make the following standard perfect cryptography assumptions (Schneier, 1996).

1. That message digests that are generated by secure hash functions are easy to compute, compress arbitrary length input to a fixed length output (the digest), are first and second pre-image resistant and are collision resistant. First pre-image resistance, or one-wayness, means that given a digest $H$ and hash function $h()$, it is computationally infeasible to find a value $x$ such that $H=h(x)$. Second pre-image resistance means that given $x$ and $h(x)$, it is computationally infeasible to find a value $y \neq x$ such that 
$h(x)=h(y)$. Collision resistance is the property that if $h(x)=h(y)$ then with effective probability of $1, x=y$.

2. That it is computationally infeasible to predict the next bit of a secure pseudo-random sequence even with complete knowledge of the algorithmic or hardware generator and of all of the previous bits in the sequence.

3. That digital signatures cannot be forged

4. That encrypted data cannot be decrypted except with the appropriate decryption key.

Digital signatures cryptographically authenticate digital information. In this chapter, we assume a digital signature scheme based on public key cryptography, where each entity has a pair of keys: one public and the other private. Public key cryptography has the following properties: (i) it is computationally infeasible to compute a private key from its corresponding public key, (ii) a ciphertext generated using a public key can only be decrypted using the corresponding private key, and (iii) a ciphertext generated using a private key can only be decrypted using the corresponding public key. It is this last property that supports digital signatures. Given a key pair and some input $x$, a digital signature scheme consists of a signing algorithm and a verification algorithm. The signer produces a signature by using their private key to encrypt $x$. The verifier users the signer's public key to decrypt $x$ and therefore verify the validity of the signature. Without loss of generality, we assumed that signature schemes are non-recoverable. That is, the verifier of a signature over input $x$ can only determine that the signature is valid. They cannot learn any other useful information about $x$ from the signature alone. In practice, a signature is typically created by using a private key to encrypt the digest of inputs as opposed to the inputs themselves. The cryptographic binding between an input $x$ and its digest, $h(x)$, means that it is effectively impossible to modify $x$ and for $h(x)$ to remain valid. Therefore a signature over $h(x)$ is treated as equivalent to a signature over $x$ and, given the fixed length of digests, it is more efficient to sign $h(x)$ than $x$.

A protocol specifies the correct behaviour of its participants. Well-behaved (honest) parties adhere to a given protocol specification. We make the following assumptions about communications between well-behaved parties and their cooperation in protocol execution.

1. The communication channel between well-behaved parties provides eventual message delivery. That is, there is a known bound on the number of temporary network and computer related failures between well-behaved parties.

2. Each well-behaved party has persistent storage for messages. Minimally, wellbehaved parties will ensure that messages are available for as long as is necessary to meet their obligations to other parties. They may require longer-term storage for their own purposes.

3. Well-behaved parties only send messages that meet the specification of the protocol being executed. Similarly, they only process messages that are correct with respect to the protocol specification.

TTPs are well-behaved parties by definition. For the purposes of this discussion, "trusted", "honest" and "correctly behaving" are synonyms of well-behaved. 


\begin{tabular}{|l|l|}
\hline Notation & Description \\
\hline$h(x)$ & secure hash of $x$ \\
\hline $\operatorname{sig}_{P}(x)$ & principal P's signature over $x$ (using P's private key) \\
\hline$e n c_{P}(x)$ & encryption of $x$ with P's public key \\
\hline$r n$ & secure pseudo-random number \\
\hline$i d_{[I]}[=f()]$ & $\begin{array}{l}\text { unique protocol run identifier with optional index } i \text { to } \\
\text { differentiate between } 2 \text { or more identifiers and optional } \\
\text { generating function }\end{array}$ \\
\hline$x, y$ & concatenation of items $x$ and $y$ \\
\hline$p a y l o a d$ & $\begin{array}{l}\text { arbitrary application data sent as payload of a protocol } \\
\text { message (for example, the original business message) }\end{array}$ \\
\hline$V A L \mid I N V A L$ & $\begin{array}{l}\text { signifies validity or invalidity of application level business } \\
\text { message }\end{array}$ \\
\hline$P \rightarrow Q:\{x, y \ldots\}$ & P sends message containing items $\{x, y \ldots\}$ to Q \\
\hline
\end{tabular}

Table 2: Protocol notation

Table 2 defines the notation used in protocol descriptions. Other, more complex tokens can be generated from the elements indicated. We assume that a protocol run identifier, $i d$ is unique for an interaction context. That is, $i d$ need not be globally unique but must be sufficiently unique to unambiguously distinguish between different protocol runs in a given context. The protocol run identifier may be an opaque value that simply satisfies this uniqueness property. Alternatively, it is common in non-repudiation protocols to cryptographically bind a protocol run identifier to other elements of protocol messages. Then, as indicated in Table 2, the identifier may be defined by some generating function. For example, the identifier may be generated from a secure hash of a random number ( $i d=$ $h(r n))$. In this case, $i d$ can be used as a commitment to the random number, $r n$, that can in turn act as an authentication token in a protocol. We assume that the protocol initiator is responsible for generation of an identifier. We also assume that if an identifier is not unique then it will be rejected by the recipient, triggering re-start of a protocol with a newly generated identifier.

\section{Voluntary non-repudiable exchange}

In a voluntary non-repudiable exchange, there is no mechanism to enforce the provision of non-repudiation information. This is the approach adopted by current commercial solutions (see BEA's WebLogic TPIE) and envisaged by Reactivity's proposed standard for Web services non-repudiation (Gravengaard, Goodale, Hanson, Roddy, \& Walkowski, 2003). In essence, to achieve voluntary non-repudiation each party simply signs the messages that they send.

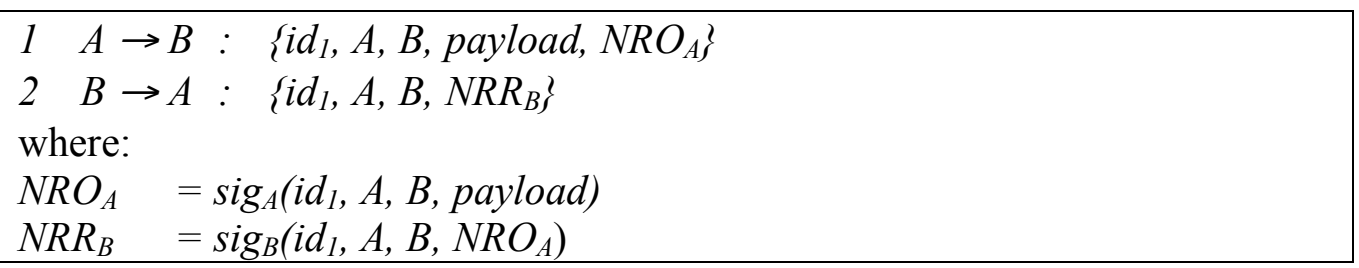

Figure 5: Voluntary non-repudiation protocol

Figure 5 shows a voluntary non-repudiation protocol for the interaction in Figure 1a. In step 1 , A sends B the business message payload along with a protocol run identifier $i d_{l}$, the participant identifiers and their NRO token. The NRO token $\left(N R O_{A}\right)$ consists of A's signature over the other items they send. Assuming B verifies the information sent by A, in step 2 they 
return a receipt message consisting of the protocol run and participant identifiers, and their NRR token. The NRR token $\left(N R R_{B}\right)$ consists of B's signature over the other items they send.

The preceding protocol is voluntary because there is no guarantee that $\mathrm{B}$ will send the receipt to A in step 2 - the selective receipt problem. A protocol of this type is suitable if the business message recipient ( $\mathrm{B}$ in this case) is trusted to provide a receipt. For example, this approach could be used for non-repudiation of the storage of the simResults document in Figure 3. In that scenario, TSP is a trusted entity that will provide a receipt to A.

Now, recall the validated message exchange shown in Figure 1b. A sends a business message to B for which B returns an ack. Subsequently, B signals the validity, or otherwise, of the message and A returns an ack for B's validation message. If we treat the validation information generated by B in the same way as business message payload, then we can use two correlated runs of the protocol in Figure 5 to make a validated exchange non-repudiable. Figure 6 shows this extension. The first run (steps 1.1 and 1.2) is identical to the exchange in Figure 5. In the second run (steps 2.1 and 2.2), B provides their validation information along with non-repudiation of validation $\left(N R O V_{B}\right)$ and A provides non-repudiation of receipt of validation $\left(N R R V_{A}\right)$. The protocol run identifier, $i d_{1}$, is included and signed in steps 2.1 and 2.2 to correlate the two runs of the voluntary protocol. Again, the exchange suffers from the selective receipt problem. That is, A must trust B to provide the receipt in step 1.2 and B must trust $\mathrm{A}$ to provide the receipt in step 2.2. If $\mathrm{B}$ does not require a receipt, and is able to perform immediate validation of A's original message, then the validated exchange can be simplified to a version of the two-step protocol of Figure 5. In this case, B appends the validation information and $N R O V_{B}$ to the second message. A protocol of this type could be used for validation on storage of the simResults document in the example application scenario.

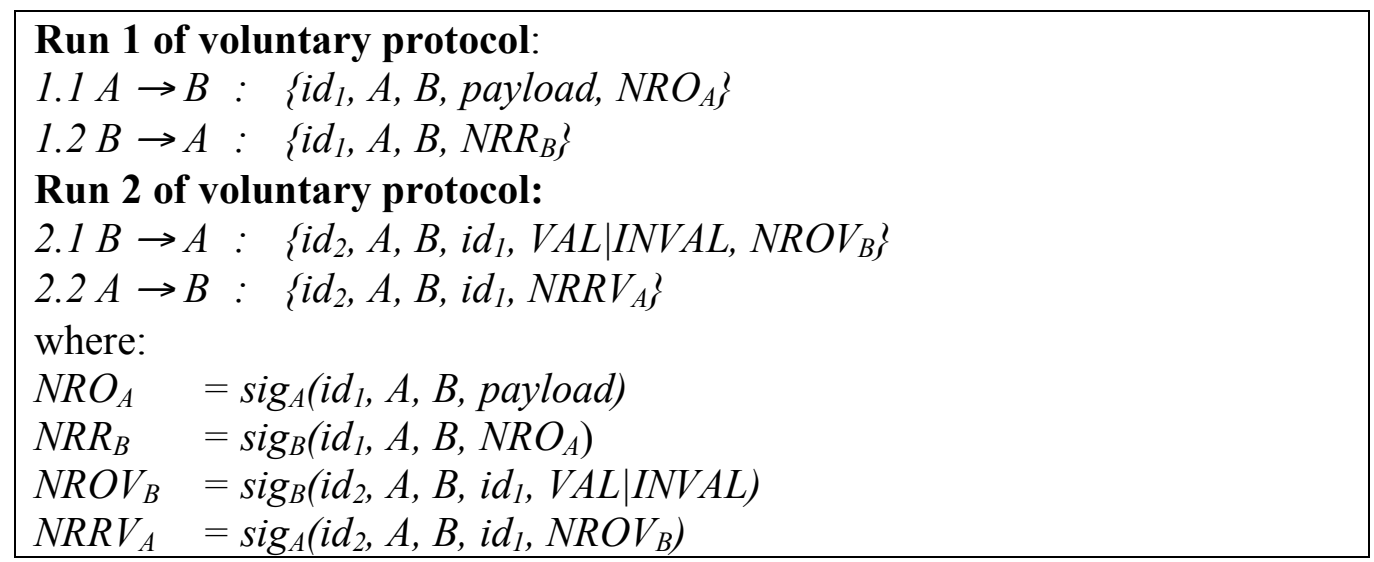

Figure 6: Voluntary non-repudiable exchange with validation

\section{Fair non-repudiable exchange}

We now show how to use fair exchange protocols to make business interactions nonrepudiable. We describe a family of protocols that use an in-line TTP. The exchange endpoints only communicate through the TTP who is then able to guarantee fairness to wellbehaved parties. The protocols are based on a verified fair non-repudiation protocol developed by Coffey and Saidha (Coffey \& Saidha, 1996) with subsequent improvements by Zhou and Gollmann (Zhou \& Gollmann, 1997). After describing this basic protocol, we introduce our modifications to the protocol to support lightweight endpoints and to integrate application-level message validation. We also define sub-protocols for timely termination of an exchange that guarantee fairness in the event of the non-cooperation, or misbehaviour, of an endpoint. 
Fair exchange with in-line TTP is well suited to a service oriented environment in which there is some trusted hub for communication between business partners. For example, the TSP in the chemical development scenario could provide such a service. However, there will be scenarios in which no sufficiently trusted hub exists or the use of the hub for every nonrepudiable exchange is too costly. In such cases, it is useful to only engage the services of a TTP when the misbehaviour of a business partner (or its endpoint) threatens to compromise fairness for a well-behaved party. There has been significant research on optimistic (off-line TTP) fair exchange protocols that address the preceding requirements. In an optimistic exchange, communicating parties exchange evidence directly and only involve a TTP in the event of non-cooperation. After the discussion of fair exchange with in-line TTP, we briefly describe the most efficient protocol for optimistic fair exchange. We also explain how to use the protocol to integrate message validation.

\section{Fair exchange with in-line TTP}

The aim of the Coffey-Saidha protocol is for the sender (A) of a business message, or payload in the notation, to obtain non-repudiation of receipt for the message from the recipient (B). Figure 7 shows normal execution of the main protocol. It is followed by a stepby-step commentary.

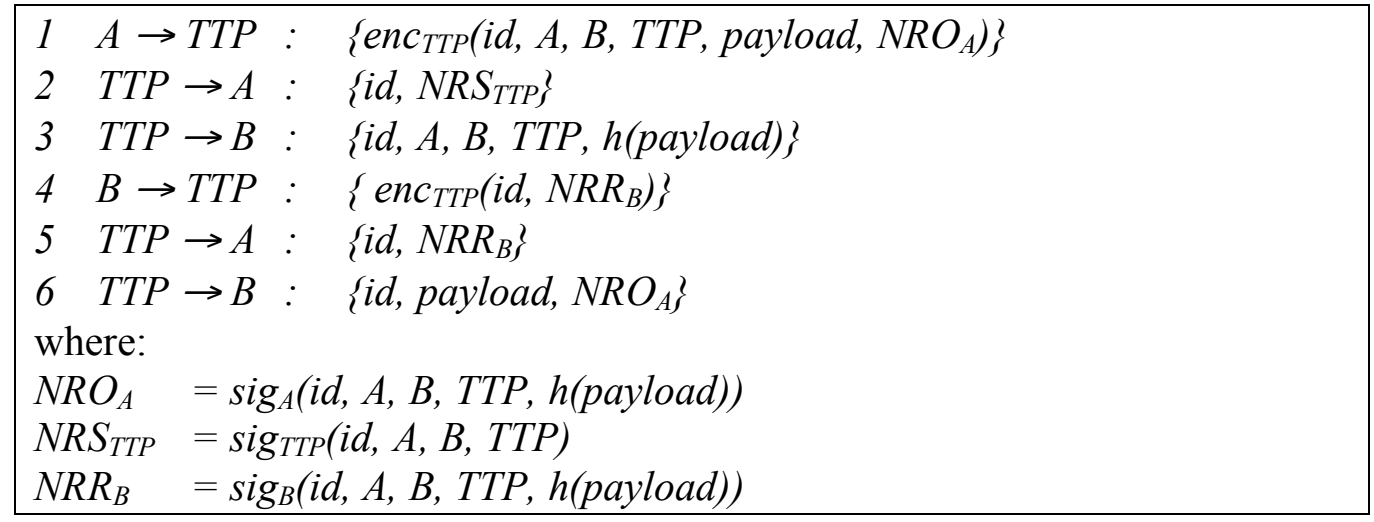

Figure 7: Main Coffey-Saidha protocol

Step 1: A starts the protocol by sending protocol run and participant identifiers, the business message payload and their NRO token to the TTP. All items are encrypted with the TTP's public key to ensure that B does not obtain the items before providing non-repudiation of receipt. At this step, if the TTP finds that the id is not unique and is unable to continue with the protocol, an appropriate response is sent to A to prompt restart of the protocol with a newly generated $i d$. Otherwise, the protocol proceeds to step 2

Step 2: The TTP provides proof of submission $\left(N R S_{T T P}\right)$ to A to signal the TTP's willingness to proceed with protocol execution. We added this step to the protocol to give $\mathrm{A}$ an immediate assurance that the exchange will commence. $N R S_{T T P}$ is sufficient for this purpose but does not constitute a receipt for the payload because only the identifiers are signed. This step may be executed in parallel with step 3 .

Step 3: To enable B to construct $N R R_{B}$, the TTP sends the identifiers and $h$ (payload) to B. This is sufficient information for $\mathrm{B}$ to be able to generate a receipt but does not give $\mathrm{B}$ access to the payload.

Step 4: B responds with $N R R_{B}$. It is safe for B to send the receipt to the TTP before obtaining the payload because the TTP is trusted to provide the payload in return. All items are encrypted with the TTP's public key to ensure that A can only obtain the receipt if the exchange of evidence terminates successfully. 
Step 5: The TTP relays $N R R_{B}$ to A. This step may be executed in parallel with step 6 .

Step 6: The TTP sends the payload and associated $N R O_{A}$ to B.

At the end of execution of the protocol, A has acquired $N R S_{T T P}$ and $N R R_{B}$ - non-repudiation of submission and receipt. In return, B has acquired payload and $N R O_{A}-$ the business message payload and non-repudiation of its origin. The TTP can guarantee fairness of the exchange because they control the release of information to A and B. On successful completion of the protocol, the TTP sets the termination state of the exchange to SUCCEEDED.

\section{Timely termination of exchange}

In order to achieve timely termination of an exchange, and at the same time preserve fairness, A or B may request that the TTP terminate execution of the main protocol in Figure 7. In this case, an abort or resolve sub-protocol is executed between the endpoint and the TTP. It is the TTP's responsibility to determine from the state of an exchange whether it can be aborted or whether it must be completed (resolved). Step 4 is the pivotal point of the protocol. Once the TTP has received $N R R_{B}$ in step 4, they have all the information necessary to complete the exchange. Prior to this point, non-cooperation by B means that the exchange can only be aborted. After this point, the TTP releases critical information to A and B (in steps 5 and 6) and the TTP must complete the exchange for any well-behaved party. A or B initiate an abort or resolve sub-protocol by sending a request to the TTP. We define the abort sub-protocol in Figure 8.

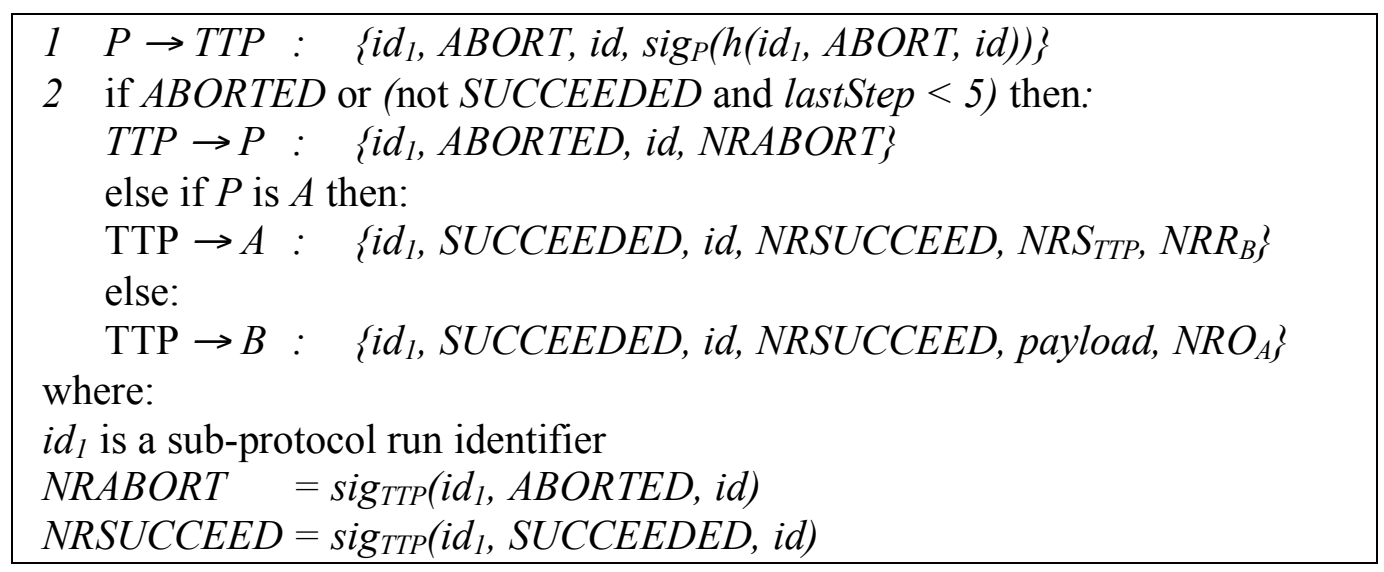

Figure 8: Coffey-Saidha abort sub-protocol

The abort sub-protocol starts with a signed abort request from one of the endpoint participants $(\mathrm{P} \in\{\mathrm{A}, \mathrm{B}\})$. To correlate the request with the exchange to which it relates, the request includes a new protocol identifier, $i d_{l}$, and the identifier of the associated main protocol, id. The TTP checks the local exchange status that consists of the exchange termination state and the number of the last step executed in the main protocol (lastStep). As shown, the exchange can be aborted if the exchange termination state is already ABORTED or the termination state has not already been set to SUCCEEDED and the main protocol has not progressed beyond step 4. If the exchange can be aborted, the TTP sends a message that signals aborted exchange to the sub-protocol initiator. This message includes non-repudiation of abort token (NRABORT) signed by the TTP. If not already set, the TTP now sets the termination state to $A B O R T E D$. If the exchange cannot be aborted (because the termination state is already SUCCEEDED or the main protocol has progressed beyond step 4), then the TTP returns a participant-specific resolve message. This message signals successful completion of the exchange with a signed NRSUCCEED token and provides the items that the participant expects from the exchange. For $\mathrm{A}$, the expected items are $N R S_{T T P}$ and $N R R_{B}$. 
For B, the expected items are payload and $N R O_{A}$ If not already set, the TTP now sets the termination state to $S U C C E E D E D$. The corresponding resolve sub-protocol is the same except that: (i) the initial message is a resolve request $\left(=\left\{i d_{1}, R E S O L V E, i d, \operatorname{sig}_{P}\left(i d_{l}\right.\right.\right.$, RESOLVE, id) $\}$ ) and (ii) the TTP only responds with an abort token if the exchange cannot be completed successfully because termination state is already $A B O R T E D$ or the main protocol has not reached step 4 yet.

Once the TTP has set termination state, either following normal termination of the main protocol or after execution of an abort or resolve sub-protocol, the TTP will forever respond in the same way to any subsequent termination request.

\section{Modifications for lightweight endpoints}

Up to now we have assumed that endpoint processing environments are such that they are able to verify each other's signatures (have access to and can verify each other's public key certificates) and that they have facilities for secure long-term storage of non-repudiation evidence. However, the TTP's mediator role allows us to adapt the Coffey-Saidha protocol to support more lightweight endpoint environments. The TTP can take on responsibility for endpoint signature verification and long-term storage of evidence. To achieve this, in steps 5 and 6 the TTP does not simply relay the NRO and NRR token provided by A and B. Instead, the TTP produces its own token:

$$
N R R_{T T P}=N R O_{T T P}=\operatorname{sig}_{T T P}(i d, A, B, T T P, h(\text { payload }))
$$

and sends this to $\mathrm{A}$ in place of $N R R_{A}$ and to $\mathrm{B}$ in place $N R O_{A}$. This replacement serves as the TTP's guarantee that they have seen, verified and will store all related evidence, including $N R O_{A}$ and $N R R_{B}$, for future reference. It essentially certifies that the TTP can prove both nonrepudiation of origin and non-repudiation of receipt of the payload. This reduces the verification work that $A$ and $B$ have to perform to that of verifying the signature and associated credentials of the TTP. This means that exchange endpoints may only require credential management at the level used by the typical Web browser rather than access to the more comprehensive PKI infrastructure required to verify evidence from all parties that they may interact with. Further, A and B only need provide long-term storage to correlate a business message exchange with non-repudiation evidence that the TTP holds in long-term secure logs. Of course, a corresponding asymmetric modification to the protocol can be made if only one of A or B is lightweight and the other is able to process all non-repudiation evidence.

Corresponding changes are made to abort and resolve sub-protocols to provide the items that $\mathrm{A}$ and $\mathrm{B}$ expect from a lightweight exchange.

\section{Extension for payload validation}

Neither of the above versions of the Coffey-Saidha protocol provides non-repudiable validation of payload. As with voluntary exchange, we could support non-repudiation of validation by correlating two runs of the protocol. However, the presence of an in-line TTP means that a more efficient extension is possible. The extension adds three extra steps to the protocol to integrate application-level validation of payload. A secure pseudo-random number $(r n)$, generated by A is used as an authenticator for the TTP to acknowledge B's validation decision. Figure 9 shows the extended protocol. It is followed by a discussion of the modifications to integrate validation. 


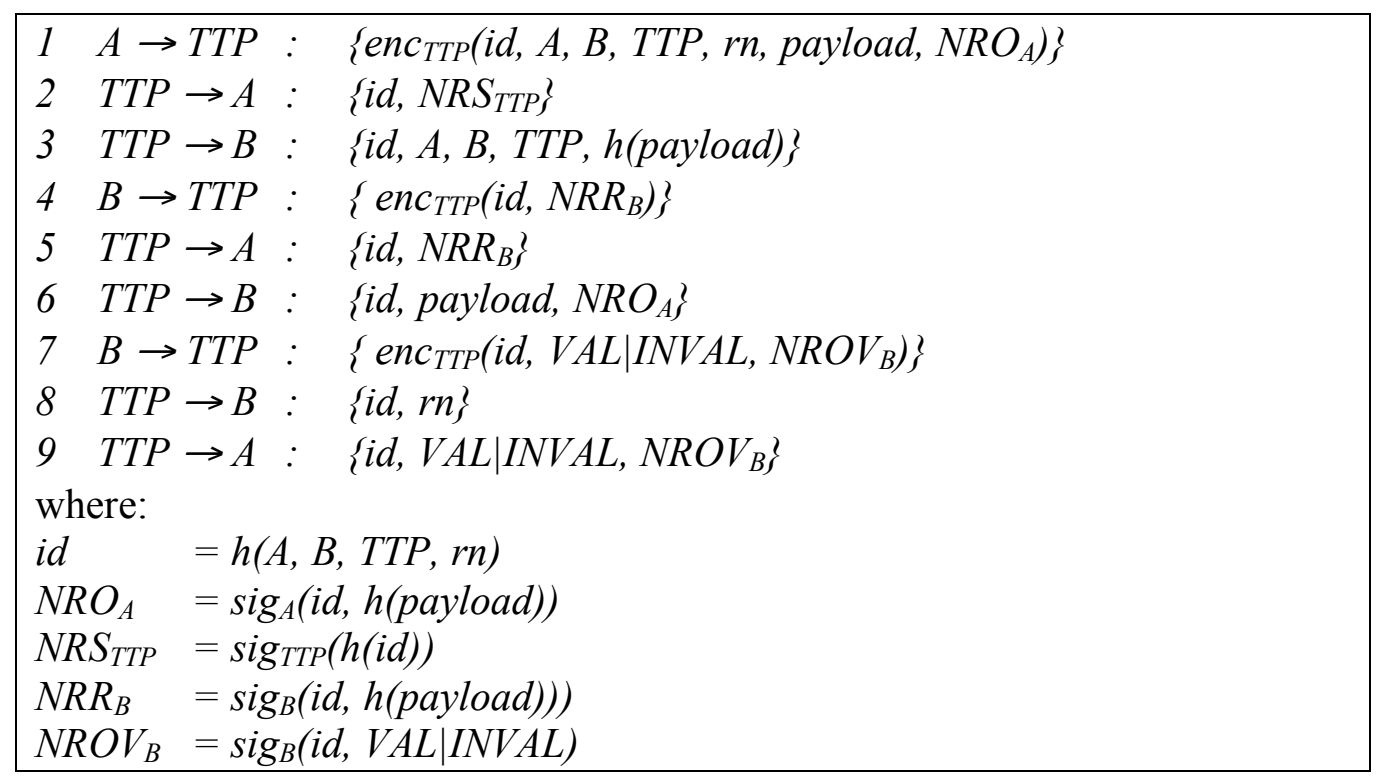

Figure 9: Extended Coffey-Saidha protocol for validation of payload

In step 1 , in addition to the items provided in the original protocol, A send $r n$ to the TTP. There is now a generating function for the protocol run identifier, $i d$. This function is a hash of the participant identifiers with $r n$. Because the TTP receives all the items necessary to verify the integrity of $i d$ in step 1, B can also rely on its integrity. Thus, $i d$ can subsequently be used by B to verify the authenticity of $r n$. As shown, because id is cryptographically bound to the participant identifiers, it is only necessary to sign $i d$, and not the other identifiers, when generating the non-repudiation tokens. Steps 2 to 6 of the protocol remain the same. Then in step 7, B sends their validation message and $N R O V_{B}$ token to the TTP. In step 8, the TTP acknowledges receipt of the validation message by sending $r n$ to B. Up to this point $r n$ was only known to A and the TTP and, as noted, it is cryptographically bound to $i d$. $r n$ therefore represents an authenticated acknowledgement for B's validation message and serves the same purpose as the $N R R V_{A}$ token from the protocol shown in Figure 6. In step 9, the TTP relays B's validation message to A. This completes the successful exchange of payload, a decision on its validity and associated evidence of the origin and receipt of messages. As for the Coffey-Saidha protocol without validation of payload, this extended version can be adapted to support lightweight endpoints. In this case, the TTP sends $N R O V_{T T P}=\operatorname{sig}_{T T P}(h(i d, V A L \mid I N V A L))$ in place of $N R O V_{B}$.

There are corresponding extensions to the abort and resolve sub-protocols to provide fair timely termination of an exchange. Details are published in (Cook et al., 2006).

\section{Optimistic fair exchange with off-line TTP}

We now use Wang's protocol (Wang, 2005) as an example of how to perform optimistic, fair, non-repudiable exchange of a message for its acknowledgement. The protocol is of interest because it is the most efficient of the existing deterministic fair exchange protocols. Again, A wishes to send business message payload to B and in return B should provide a receipt. In the protocol, A first sends receipt input information for B to sign in order to generate a receipt. B then sends the receipt to A and then A sends the payload to B. The key insights behind the protocol are:

1. That A's interests are safeguarded because they obtain a receipt for the payload before sending the payload to B. 
2. B's interests can be safeguarded if the receipt input is cryptographically bound to the payload through A's evidence of origin and, in the event of A ceasing cooperation, a TTP, and only the TTP, can enable B to recover the payload from the receipt input.

\begin{tabular}{lll|}
\hline$A \rightarrow B:$ & $\left\{i d, A, B, T T P\right.$, payloadCipher, $h(k A)$, keyCipher, $\left.N R O_{A}\right\}$ \\
$2 B \rightarrow A:$ & $\left\{i d, N R R_{B}\right\}$ \\
$3 A \rightarrow B:$ & $\{i d, k A, r n\}$
\end{tabular}

Figure 10: Wang's optimistic fair exchange protocol

Figure 10 shows the main exchange protocol. To start the protocol A generates two encrypted tokens: payloadCipher and keyCipher. payloadCipher is the encryption of the payload using the secret key $k A$ that is known only to A. keyCipher is the encryption of the secret key with the protocol identifier and a random number using the TTP's public key. The id is cryptographically bound to the payload through the digest of the payloadCipher used in construction of the $i d$. Only the TTP can decrypt keyCipher but any other party can verify that it is an encryption of $i d$ and $k A$ with some random number. A sends the encrypted items to $\mathrm{B}$ along with participant identifiers, including the identifier of the off-line TTP, and $N R O_{A}$. $N R O_{A}$ cryptographically binds keyCipher to id and therefore to payloadCipher and ultimately to payload. Given this message, B can generate a receipt by signing the same items as A to produce $N R R_{B}$. Now, B knows that either: (i) A prepared keyCipher correctly and, therefore, that A or the TTP can recover $k A$ and $r n$ from keyCipher to enable $\mathrm{B}$ to verify NRO evidence and obtain payload; or (ii) A provided an invalid keyCipher and therefore $N R R_{B}$ will not be a valid receipt. That is, B's receipt is only valid if A provides valid input that, if necessary, $\mathrm{B}$ can use to recover the payload from the TTP. Therefore, it is safe for $\mathrm{B}$ to send $N R R_{B}$ to $\mathrm{A}$ in step 2. In the final step, A sends B $k A$ and $r n$. These items allow B to verify the integrity of all evidence previously provided by A and to decrypt payloadCipher to obtain payload.

A full discussion of the security properties of the protocol, how fairness and timeliness are guaranteed, and the specification of abort and resolve sub-protocols is presented in (Wang, 2005). Here we briefly describe protection against cheating by either A or B.

A can attempt to cheat in two ways: (i) they can provide B with an invalid keyCipher or invalid payloadCipher, or (ii) they can decline to send the final message. As discussed previously, invalid input from A will lead to the generation of a useless receipt from B. Therefore, A cannot achieve non-repudiation of receipt if they provide invalid input to $B$. If A does not send the final message, then B can obtain $k A$ and $r n$ from the TTP in return for $N R R_{B}$. The TTP will provide $k A$ and $r n$ unless A has requested that the exchange be aborted. If the exchange has been aborted then the TTP provides B with a signed abort token that can be used to repudiate receipt of payload. Therefore, A cannot obtain a valid receipt unless both payload and NRO are available to B. B can only attempt to cheat by failing to provide A with a valid receipt and requesting that the TTP provide $k A$ and $r n$. However, the TTP only provides these items to $\mathrm{B}$ if $\mathrm{B}$ provides the TTP with $N R R_{B}$ and all other protocol items for the TTP to verify their consistency and integrity. Further, if the TTP enables B to recover payload they also provide A with B's valid receipt. 
In conclusion, there is no advantage to either A or B attempting to cheat and therefore the optimistic exchange is only likely to fail because of timeliness constraints. That is, in the normal case the exchange will complete as a direct exchange between $\mathrm{A}$ and $\mathrm{B}$ without the need to involve the TTP.

As with voluntary exchange, we integrate payload validation by executing two correlated runs of the protocol: one to exchange payload and NRO for NRR and the other to exchange validation decision and NROV for NRRV. The identifier of the first protocol run is used for correlation with the second.

\section{Summary and evaluation}

In this section, we have described the voluntary and fair exchange approaches to making business interactions non-repudiable. The voluntary approach is appropriate for interactions between TTPs or when the recipient is a TTP (as in phase 1 of interactions 1 and 3 of Figure 3 ). Fair exchange is appropriate for interactions between business partners (as in Interaction 2 of Figure 3). In the case of fair exchange with in-line TTP, we have also demonstrated how to adapt a protocol for interactions between lightweight participants.

The protocols presented up to now address the following classes of interaction: TTP to TTP, business partner to TTP and business partner to business partner. The remaining class of interaction is TTP sender to business partner recipient. In this case, to guarantee fairness, it is necessary to ensure that the TTP obtains a receipt regardless of the behaviour of the other party. To achieve this we merge the roles of A and the TTP in the Coffey-Saidha protocol. This reduces the six-step protocol in Figure 7 to the three steps shown in Figure 11. In this protocol, A is a TTP and solicits a receipt for the payload before sending the payload to B. This protects A's interests because they only release the payload if B provides a valid receipt. B's interests are protected by the fact that A is trusted to send message 3 . For timely termination, A can unilaterally abort the exchange before B sends the second message. After step 2, the exchange is guaranteed to complete. Validation can be integrated by adding two steps for B to send their validation message and A to return a receipt, which they are guaranteed to do because they are a TTP.

$$
\begin{aligned}
& 1 \quad A \rightarrow B: \quad\{i d, A, B, h(\text { payload })\} \\
& 2 \quad B \rightarrow A:\left\{i d, N R R_{B}\right\} \\
& 3 \quad A \rightarrow B: \quad\left\{i d, \text { payload, } N R O_{A}\right\} \\
& \text { where: } \\
& N R O_{A}=\operatorname{sig}_{A}(i d, A, B, h(\text { payload })) \\
& N R R_{B}=\operatorname{sig}_{B}(i d, A, B, h(\text { payload }))
\end{aligned}
$$

Figure 11: TTP sender to business partner recipient receipt-first exchange

The choice of which exchange mechanism to use will depend on the application context. For example, as discussed up to now, the relationships between protocol participants will have an influence on the choice. When choosing between in-line TTP and off-line TTP approaches to fair exchange, the availability and the capabilities of TTP services may be significant factors. An obvious consideration is the communication overheads of a protocol in terms of the number of messages needed to achieve the exchange of evidence. However, care must be taken when calculating these overheads. If signing keys are revocable and trusted timestamps are required for signature verification and the long-term validity of evidence, then the costs of obtaining time-stamps from a TSA must be included in the calculation. 


\begin{tabular}{|l|c|c|c|}
\hline Approach & $\begin{array}{c}\text { Protocol } \\
\text { messages }\end{array}$ & $\begin{array}{c}\text { Time- } \\
\text { stamp } \\
\text { messages }\end{array}$ & $\begin{array}{c}\text { Total } \\
\text { messages }\end{array}$ \\
\hline $\begin{array}{l}\text { (ii) In-line TTP fair exchange (Coffey- } \\
\text { Saidha - Figure 7) }\end{array}$ & 6 & 6 & 12 \\
\hline $\begin{array}{l}\text { (iii) In-line TTP/TSA fair exchange } \\
\text { (Coffey-Saidha) }\end{array}$ & 6 & 0 & 6 \\
\hline $\begin{array}{l}\text { (iv) Off-line TTP fair exchange (Wang- } \\
\text { Figure 10) }\end{array}$ & 3 & 4 & 7 \\
\hline
\end{tabular}

Table 3: Communication costs of fair exchange

Table 3 compares the costs of three approaches to business partner to business partner fair exchange of a message for its receipt: (i) with an in-line TTP, (ii) with an in-line TTP that is also a TSA, and (iii) with an off-line TTP for Wang's optimistic protocol. It should be noted that it is not possible to optimise optimistic fair exchange when a TTP also acts as TSA since there is no TTP involvement in the main exchange. Column 2 shows the number of nonrepudiation protocol messages in each case. In terms of protocol messages, the off-line TTP fair exchange is cheapest with just three messages. Column 3 shows the number of messages required for trusted time-stamping. In each protocol, a time-stamp must be obtained the first time that signed evidence is generated. Two messages are required for each time-stamp, one to send the request to the TSA and the other for the TSA's response. In the first case, three of the in-line TTP protocol messages require time-stamping and this leads to an overhead of six messages. As shown, there is no overhead in the second case because the TTP is also a TSA and performs all time-stamping. The off-line TTP protocol requires a time-stamp for two of its three messages, incurring an overhead of four messages. As shown in the final column, fair exchange with an in-line TTP/TSA is now cheaper, in terms of total messages required, than the off-line TTP approach. That is, the relative communication costs of the different approaches change when time-stamps from a TSA are required.

\begin{tabular}{|l|c|c|c|}
\hline Approach & $\begin{array}{c}\text { Protocol } \\
\text { messages }\end{array}$ & $\begin{array}{c}\text { Time- } \\
\text { stamp } \\
\text { messages }\end{array}$ & $\begin{array}{c}\text { Total } \\
\text { messages }\end{array}$ \\
\hline $\begin{array}{l}\text { (ii) In-line TTP fair exchange with } \\
\text { validation (Coffey-Saidha - Figure 9) }\end{array}$ & 9 & 8 & 17 \\
\hline $\begin{array}{l}\text { (iii) In-line TTP/TSA fair exchange with } \\
\text { validation (Coffey-Saidha) }\end{array}$ & 9 & 0 & 9 \\
\hline $\begin{array}{l}\text { (iv) Off-line TTP fair exchange with } \\
\text { validation (Wang) }\end{array}$ & 6 & 8 & 14 \\
\hline
\end{tabular}

Table 4 Communication costs of fair exchange with application-level validation

Table 4 compares communication costs when the exchange includes application-level validation of the business message. As shown, when time-stamping is required, the in-line TTP/TSA fair exchange is now significantly cheaper than the off-line TTP approach. In fact, it is cheaper than the voluntary exchange approach shown in Figure 6 that incurs an overhead of eight messages for its four protocol messages (assuming a separate TSA is required and validation cannot be combined with receipting). Clearly, if communication costs are a factor in the choice of mechanism then important considerations include whether trusted timestamping is required and whether the guarantor TTP can also provide the TSA service. Other factors, such as computational costs, will also inform the choice of mechanism. 
The preceding analysis, and the choice of protocols presented in this section, underlines the desirability of being able to choose an appropriate non-repudiation mechanism to suit the given B2B context. The flexibility necessary to meet this challenge is based on the model of interceptor-mediated interaction described in this section. Our framework for protocol execution between and with Web services is a practical realisation of the model. The execution framework is sufficiently flexible to allow partners to choose to execute a protocol appropriate to the sort of interaction they are engaged in on a per business message basis. It also supports arbitrary, application-level validation of business messages.

\section{WEB SERVICES PROTOCOL EXECUTION FRAMEWORK}

In this section, we present our Web services based implementation of a framework for nonrepudiation protocol execution (WS-NRExchange). A prime requirement on this middleware is that it is able to support the execution of any deterministic non-repudiation protocol. It achieves this flexibility through: (i) a well-defined, generic interface for the exchange of protocol messages between protocol participants, (ii) an extensible schema that defines the content of self-describing protocol messages, and (iii) a well-defined API for message processing by the middleware that includes the registration of protocol-specific handlers for messages.

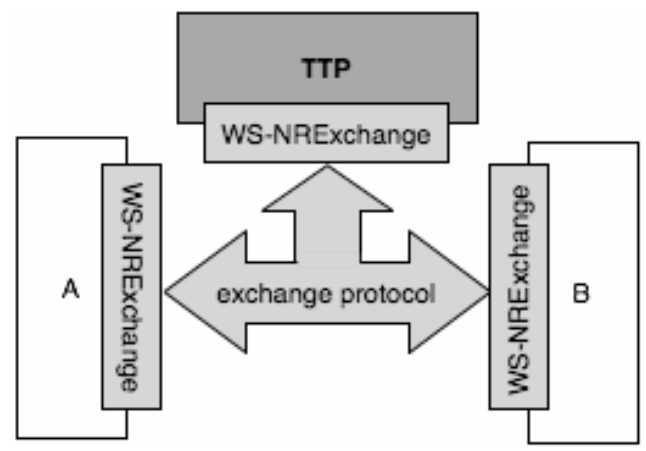

Figure 12: WS-NRExchange mediated interaction

As illustrated in Figure 12, the middleware is a realisation of the mediated interaction model described in the previous section. Each protocol participant, including any guarantor TTP, exposes a WS-NRExchange endpoint for protocol execution. At business partners (A and B in Figure 12), the WS-NRExchange middleware intercepts B2B Web service messages. This allows the middleware to adapt to different application requirements, including the execution of different protocols, without disturbing application-level logic. For example, the middleware can execute any of the protocols described in the previous section. We have demonstrated this approach in the context of the Gold project (Periorellis et al., 2006). We successfully applied non-repudiation and application-level validation to the exchange of SOAP messages between and with existing Web services without modification to the services or their clients. We used AXIS Web services deployed in the Tomcat container (Apache Software Foundation, 2005a, 2005b) as the demonstrator platform. However, apart from some easily isolated AXIS-specific interceptor code, the WS-NRExchange middleware is AXIS-independent and could be re-deployed on a range of different Web services platforms. This includes deployment as part of a separate mediation service that is not co-located with the applications to which non-repudiation is applied.

In this section, we present a general overview of WS-NRExchange interaction and infrastructure services. Then we describe the generic Web service interface to protocol message exchange and the associated message schema. This includes the ability to instantiate 
protocol-specific handlers at runtime in order to adapt the middleware to the use of different protocols as appropriate for the given interaction. First, we provide an overview the standards used to support WS-NRExchange and discuss the limitations of the XML Signature standard when expressing certain elements of non-repudiation protocol message.

\section{Overview of Web services and supporting standards}

Figure 13 shows how various XML and Web service standards support WS-NRExchange. SOAP is the basic messaging protocol for communication with and between Web services. We use the SOAP header for message processing information, including non-repudiation protocol elements. The SOAP body carries the business message payload.

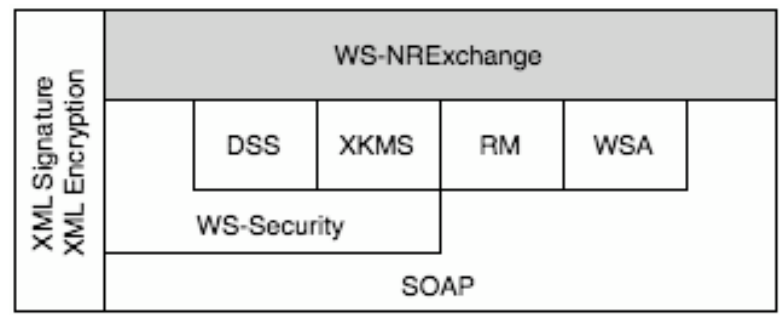

Figure 13: WS-NRExchange and Web service standards

The WS-Security standard (Nadalin, Kaler, Hallam-Baker, \& Monzillo, 2004) covers the creation of self-protecting messages for Web services. WS-Security applies XML technologies, such as XML Signature (Eastlake, Reagle, \& Solo, 2002) and XML Encryption (Eastlake, Reagle, Imamura, Dillaway, \& Simon, 2002), to SOAP messages. The XML Signature standard specifies how to generate a signature over some referenced data, how to encode the signature in XML and how to attach the signature, with related information, to XML documents. The standard also specifies how the recipient of a signature should process the XML elements in order to verify the integrity of the signature. XML Encryption is the corresponding standard for encryption. The WS-Security standard specifies a security header for SOAP messages that can convey XML Signature and XML Encryption elements. Other elements carried in the security element include authentication tokens, secure time-stamps, and credentials or certificates.

Digital Signature Service (DSS) (Perrin et al., 2004) and XML Key Management Specification (XKMS) (Hallam-Baker \& Mysore, 2005) are higher level specifications that use WS-Security. DSS specifies a service for the verification and the application of signatures to XML, and for trusted time-stamping of signed information. XKMS concerns public key life-cycle management. It specifies Web service-based PKI functionality such as how to register, locate, verify and revoke the digital credentials that are associated with public keys. XKMS and DSS may be offered as TTP services to support secure Web service interactions, thereby reducing the security infrastructure requirements of user organisations. Organisations may also provide a sub-set of the services in-house as part of their own security infrastructure. For example, an in-house DSS service can be used to apply corporate signatures to XML messages.

Reliable messaging (RM) specifies the message content, protocols and persistence requirements necessary for Web services to implement various forms of reliable message delivery. To provide at-least-once message delivery, we use an implementation of WSReliableMessaging (Bilorusets, 2005).

We use WS-Addressing (WSA) (Gudgin, Hadley, \& Rogers, 2006) for message routing and for the description of WS-NRExchange Web service endpoints. 
Our contribution is to provide the WS-NRExchange middleware that uses the identified standards. The middleware includes the specification of the NRExchange XML schema (Cook \& Robinson, 2006) for a non-repudiation protocol SOAP header, protocol implementations and protocol message processing services with associated WSDL

(Christensen, Curbera, Meredith, \& Weerawarana, 2001). As far as possible, we use the XML security standards to encode protocol tokens in XML and include those tokens in the WSSecurity header. We developed the NRExchange schema to encode tokens that either cannot be expressed using the security standards or whose expression has potential to conflict with the processing model specified by the standards. The NRExchange schema also specifies how to include protocol context and processing information in the SOAP header. Before describing the middleware, we discuss the limitations of the XML Signature standard and its processing model with respect to non-repudiation protocols.

\section{Limitations of the XML Signature standard}

XML Signature is an important standard for the inter-operable exchange of digital signature and related information. It can be used for the specification of cryptographic algorithms, references to source data, and the representation of message digests and of signatures over the digests. As noted in the preceding discussion, and as importantly, the standard also specifies a processing model for the generation and verification of signatures. Digital signatures and message digests are an integral part of non-repudiation protocols. Therefore, the standard is a fundamental building block for the XML representation of non-repudiation protocol messages and the processing of those messages. However, the processing model has limitations when it comes to verifying information exchanged in fair exchange protocols. The problem arises because fair exchange protocols rely on the fact that signatures, and digests, provided in one message may reference information that is not made available until a later message. For example, B cannot verify the digest (h(payload)) that they receive in message 3 of Figure 7 until they receive the payload in message 6. Another example, from the protocol in Figure 9, is that A's signature over $i d$, and therefore over the included digest of $r n$, that is sent to $\mathrm{B}$ as $N R O_{A}$ in step 6 is not fully verifiable until B receives $r n$ in step 8. But, the core XML Signature processing model specifies that referenced inputs to signatures must be available for verification upon receipt of a signature.

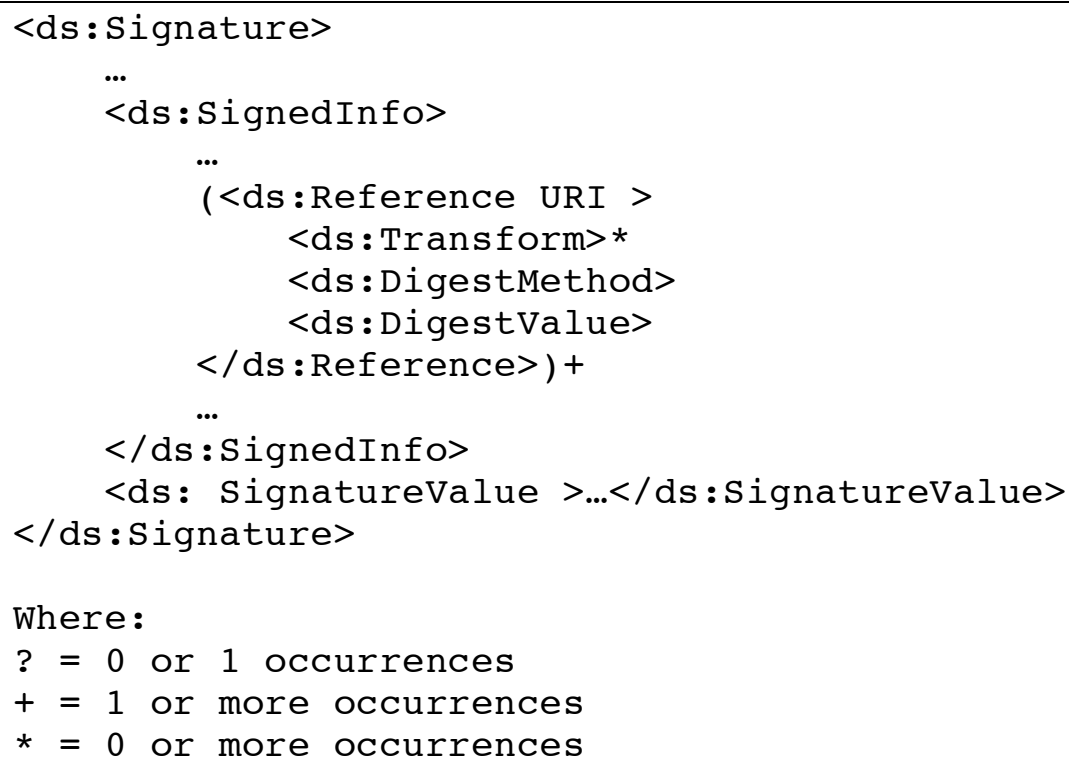

Figure 14: ds:Signature element 
As shown in Figure 14, every Signature element contains at least one SignedInfo element that in turn contains at least one Reference element. The SignedInfo element specifies the input that was used to generate the Signaturevalue. A Signature element also contains other information such as the method used and the signer's certificate. The inputs for signing are a sequence of message digest values specified in the SignedInfo Reference elements. A Reference element specifies a URI to the source data, zero or more transformation methods to apply to the data (specified by the Transform element), the digest method applied to the transformed data and the digest value that is used as input to signature generation. The XML Signature processing model specifies that the source data identified by the Reference URI must be available to the processor of the Signature. That is, the recipient processor of a Signature must be able to de-reference all the Reference element URIs, apply the specified transforms to the referenced data and generate the same digest value in order to verify the integrity of the signature. As noted previously, fair exchange protocols achieve fairness by delaying the release of information. However, reference processing will fail if a Reference element that cannot be de-referenced is placed in a SignedInfo element. The XML Signature standard does specify a Manifest element that may contain one or more Reference elements. The standard allows that one or more of the Reference elements contained in a Manifest may not be de-referencable.

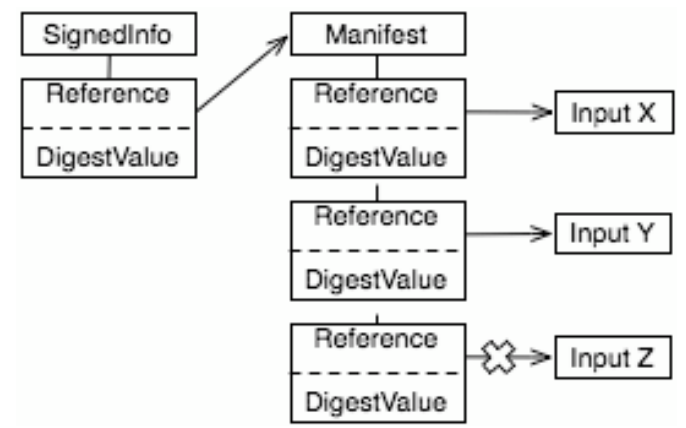

Figure 15: Indirect referencing in XML Signature

Figure 15 illustrates usage of the Manifest element. A SignedInfo element contains a Reference that references a Manifest element. The SignedInfo digest value is the digest over the whole of the Manifest element. In this example, the Manifest element contains three Reference elements that refer to inputs $\mathrm{X}, \mathrm{Y}$ and $\mathrm{Z}$. As shown, inputs $\mathrm{X}$ and $\mathrm{Y}$ are available but input $\mathrm{Z}$ is not. So, the digest value in the Reference in the SignedInfo element can be verified because it refers to the Manifest element. The Reference elements that refer to inputs $\mathrm{X}$ and $\mathrm{Y}$ can also be verified. The Reference element that refers to input $Z$ cannot be verified. This level of indirection allows the SignedInfo element to comply with the XML Signature processing model because all references in that element can be verified. The semantics for Manifest processing are application-specific. The standard allows either that the verification process may tolerate the inability to verify one or more Manifest references, or that the verification process may fail after the first failed attempt to dereference a Manifest reference. For the example in Figure 15, it is applicationspecific whether processing of the Manifest element will succeed or will fail on encountering the reference to input $\mathrm{Z}$.

To address the problem with XML Signature references, we define a MessageReference element in our schema for protocol messages. The MessageReference element supports forward and backward referencing of protocol messages. As with the Manifest element, we exploit reference indirection to preserve processing semantics. Unlike the Manifest element, our MessageReference element has unambiguous processing semantics. In addition, it supports the elegant expression of protocol tokens such as receipts and functions 
to generate protocol run identifiers. We provide a detailed description of the MessageReference element in the discussion of the NRExchange schema that follows an overview of WS-NRExchange interaction.

\section{WS-NRExchange interaction}

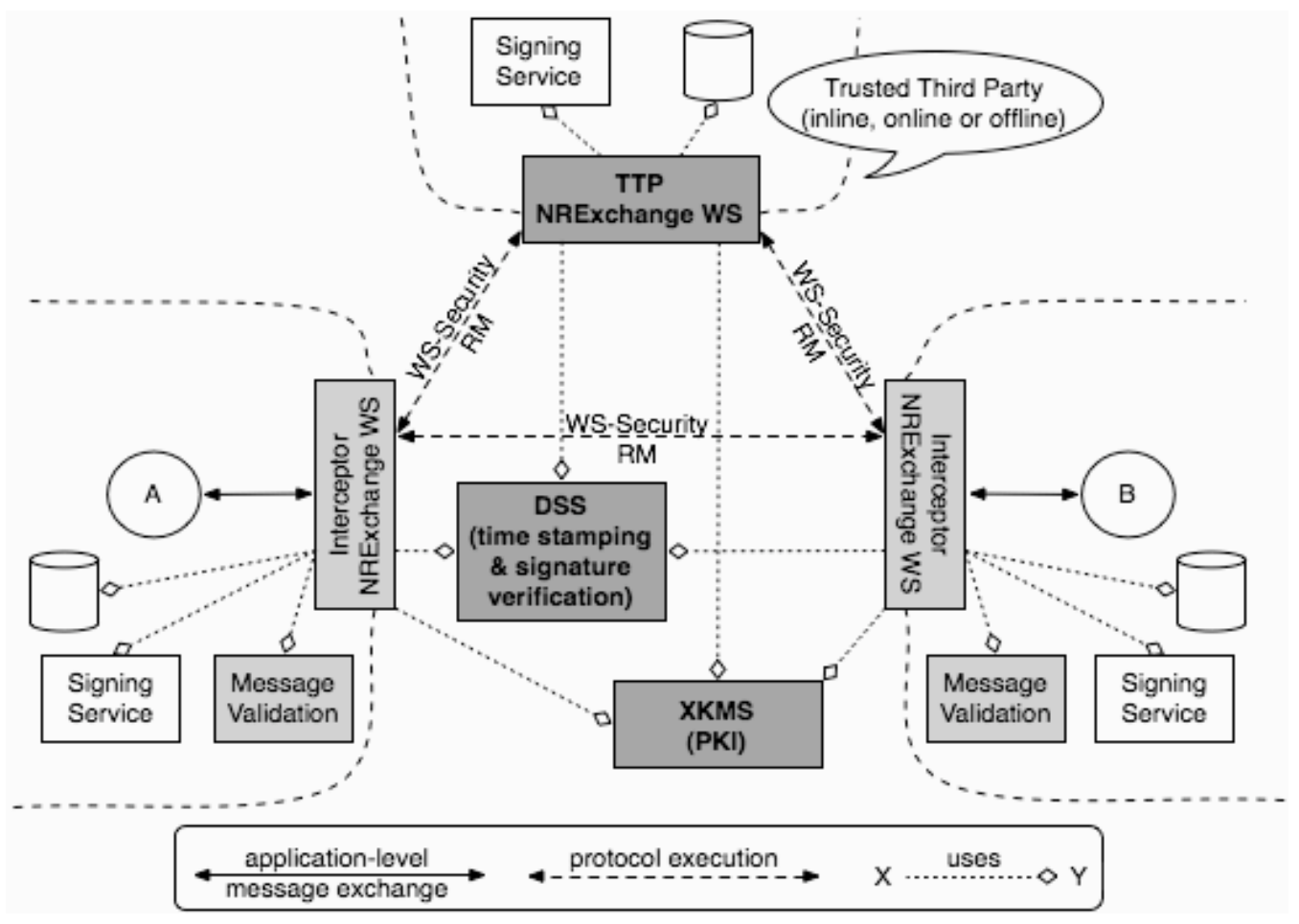

Figure 16: WS-NRExchange architecture

Figure 16 shows the interactions between the main components and services that comprise our implementation. The TTP, A and B each provides an NRExchange Web service that manages their participation in non-repudiation protocols. Each of these Web service exposes the same interface for non-repudiation protocol execution. At A and B the service is deployed as an interceptor to mediate Web service interactions that require non-repudiation. This interceptor may be co-located with the local application that uses it or, for example, may be part of a corporate firewall service. The endpoints, A and B, may themselves be Web services or Web service clients or both. The introduction of the NRExchange services does not require any modification to these application-level processes at A or B. The NRExchange services access additional local services for signing evidence, message persistence and applicationlevel validation. The signing service is required to apply signatures to the parts of messages that have not already been signed. If signatures have been applied at the application level, the NRExchange service only needs to countersign the generated signatures and not the message content. This service may be an implementation of DSS or some other mechanism for obtaining private keys to apply signatures as defined by WS-Security. Persistence is required to meet fault tolerance requirements and for audit. The NRExchange services also access trusted time-stamping services and public key management services (for example, DSS and XKMS services provided by third parties). For protocols that use an in-line TTP, trusted time-stamps may optionally be applied by the TTP Web service.

As previously noted, a WSDL interface has been defined for the interaction between NRExchange services. The SOAP messages exchanged comply with the WS-Security specification. 
The NRExchange Web service also provides a local interface to allow registration of application-specific listeners for message validation and other events. A message validation listener may trigger arbitrarily complex validation of a business message. If no validation listener is registered, then the NRExchange service assumes that a message is valid with respect to business-specific constraints. Messages that are found to be invalid are logged but are not passed to the target application for processing. Registration of event listeners allows notification of protocol-related events. For example, an application can register to receive notification of zero or more of the acknowledgements generated by the non-repudiation protocols.

\section{Generic NRExchange interface and message schema}

Figure 17 is an extract of the NRExchange Web service WSDL that shows the operations that are exposed to other NRExchange services for protocol execution.

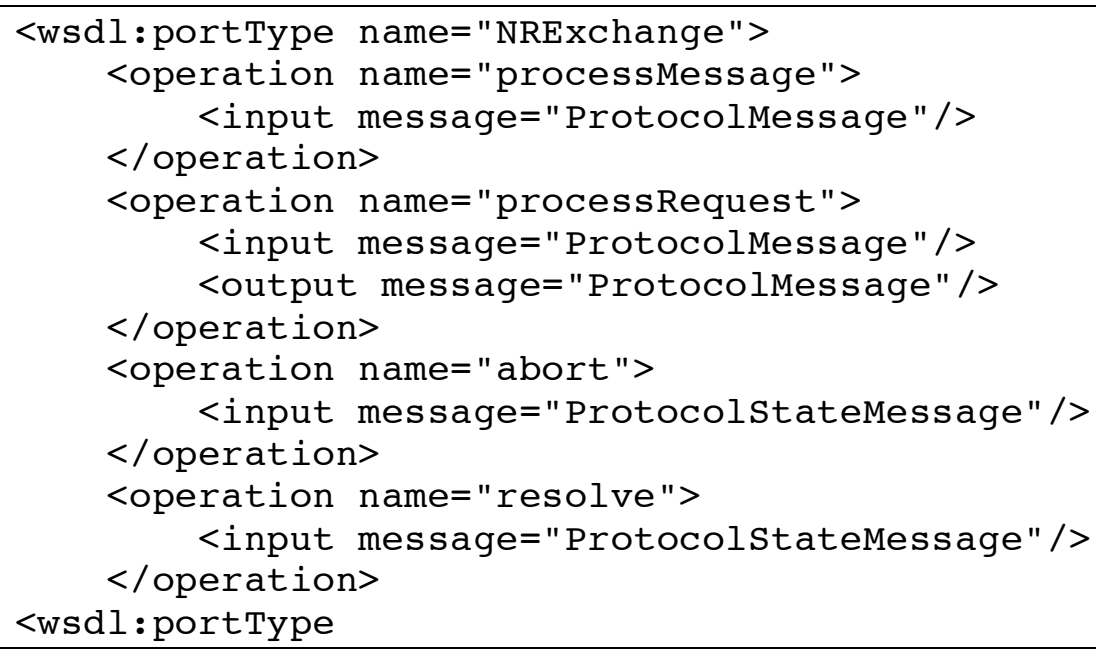

Figure 17: Extract of NRExchange WSDL

NRExchange services use the processmessage operation to exchange non-repudiation protocol messages with each other. The sender provides a protocol message for the receiver to process as specified by the given protocol. Message elements are defined in a related XML Schema. The schema is sufficiently general and extensible for the processmessage operation to be used to execute any protocol that participant services support. The processRequest convenience operation allows send and receipt of protocol messages as request/response pairs.

The abort and resolve operations are for pro-active termination. These operations are typically used by a TTP to inform another participant service that an identified exchange has been aborted or resolved with the given protocol termination state. Invocation of these operations may result in execution of a new sub-protocol using the protocol execution operations.

The SOAP binding for the NRExchange service specifies two types of message:

1. protocol messages that are exchanged during execution of a main protocol or of related sub-protocols using processmessage and, optionally, processRequest; and

2. protocol state messages that convey information about the state of an identified protocol (exchanged using abort or resolve). 
Both types of message use a WS-Security header to carry security tokens such as: signatures over evidence, time-stamps, credential and key information, security context and access control information.

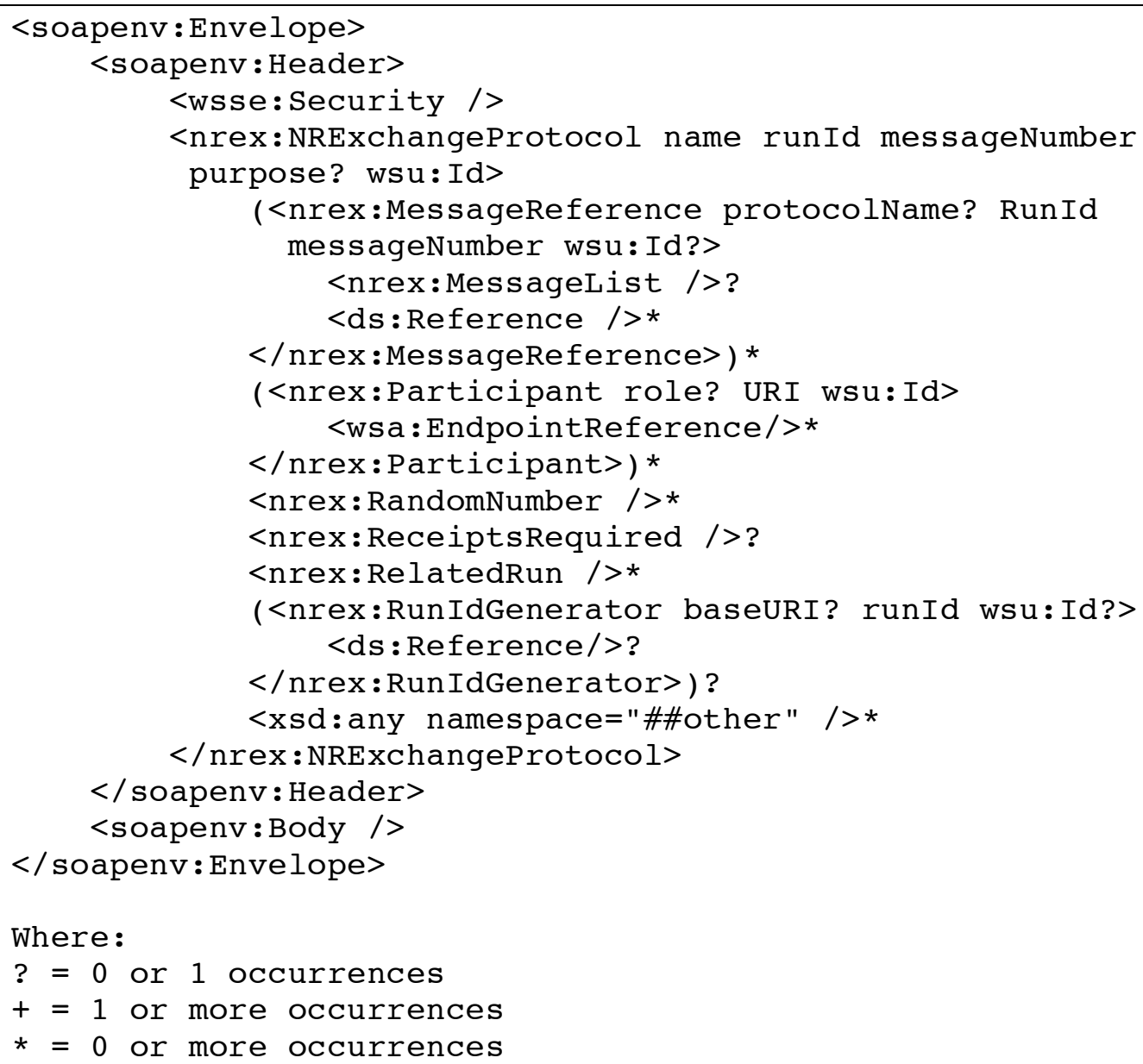

Figure 18: General form of ProtocolMessage

As shown in Figure 18, protocol messages must have an NRExchangeProtocol header. This is an extensible container for non-repudiation protocol elements. The elements are defined in the NRExchange XML schema (Cook \& Robinson, 2006). The NRExchange schema specifies that any NRExchangeProtocol header must have protocol name, runId and messagenumber attributes.

The protocol name is a URI that serves to uniquely identify the protocol, or sub-protocol, being executed. For example, Table 5 shows names for the set of protocols that together implement the Coffey-Saidha exchange without application-level validation of payload. In addition to protocols for the main exchange and abort and resolve, there is a getStatus subprotocol. This sub-protocol allows one participant to query another's view of current protocol state with the option of requesting missing messages. A getStatus protocol will involve the exchange of evidence to establish that a participant is entitled to the requested information. Implementation of getStatus sub-protocols is optional.

\begin{tabular}{|l|l|}
\hline Protocol & Name \\
\hline Main exchange protocol & $\begin{array}{l}\text { http://www.cs.ncl.ac.uk/nrex/fairex/coffey- } \\
\text { saidha/main }\end{array}$ \\
\hline Abort sub-protocol & http://www.cs.ncl.ac.uk/nrex/fairex/coffey- \\
\hline
\end{tabular}




\begin{tabular}{|l|l|}
\hline & saidha/abort \\
\hline Resolve sub-protocol & $\begin{array}{l}\text { http://www.cs.ncl.ac.uk/nrex/fairex/coffey- } \\
\text { saidha/resolve }\end{array}$ \\
\hline Get status sub-protocol & $\begin{array}{l}\text { http://www.cs.ncl.ac.uk/nrex/fairex/coffey- } \\
\text { saidha/getStatus }\end{array}$ \\
\hline
\end{tabular}

Table 5: Example protocol names

The runId is the protocol run identifier ( $i d$ in the protocol notation). The schema specifies that the runId is a URI. The runId URI may be opaque or it may have a generating function associated with it. For example, the runId in Figure 9 is generated from $h(A, B$, $T T P, r n)$. In such cases, the RunIdGenerator element can be used to specify the generating function. The RunIdGenerator specifies an optional baseURI and the function over inputs as zero or one digest Reference. A URL-safe Base64 encoding (Josefsson, 2003) of the digest value is used to construct the URI. The runId in Figure 20 is an example of URL-safe Base64 encoding of a digest value where the Base64 character "/" has been converted to a "_". When, for authentication purposes, a runId is constructed from items not available in the current message, we exploit the forward and backward referencing facility provided by the MessageReference element.

The messagenumber is a positive, non-zero value that corresponds to the step of the protocol being executed.

Depending on the protocol being executed, or the step of the protocol, the following optional items may be included in the NRExchangeProtocol header: the purpose of the protocol message (NRR, NRO etc.), the participants in the protocol, the runIds of any related protocol or sub-protocol runs, information related to receipts required, and protocol state information.

The SOAP message body contains the application payload that the business message sender intended for the ultimate recipient.

Protocol state messages do not have an NRExchangeProtocol header. The message body carries protocol state information that is intended for another NRExchange service. The general form of a protocol state message is shown in Figure 19. In addition to identifying the protocol and run to which a message relates, a Protocolstate element may include information such as: the protocol run status; if terminated, the termination state; and the message numbers of any messages seen by the recipient. Protocol messages may be provided as attachments to a protocol state message.

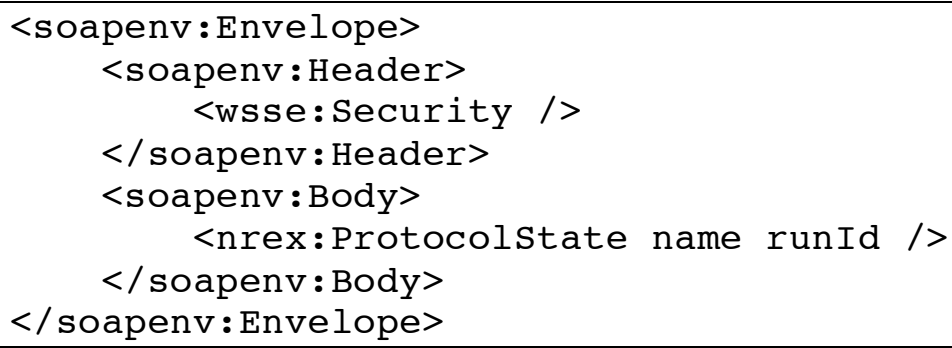

Figure 19: General form of ProtocolStateMessage

\section{Forward and backward references to messages}

Now we explain how we address the referencing limitations of the XML Signature standard discussed previously. One approach would be to use the XML Signature Manifest element in a WS-Security header and to restrict the semantics for processing the element to tolerating a failure to dereference. For our purposes, the main problem with this approach is that the 
processing semantics for the Manifest element are dependent on the meaning of the element in the context of a non-repudiation protocol that cannot be inferred from the WSSecurity header context. Furthermore, intermediate processors must respect the protocolspecific processing semantics. Therefore, as shown in Figure 18, we have defined our own MessageReference element that is a wrapper for zero or more XML Signature Reference elements.

The MessageReference provides a mechanism to reference both existing and future protocol messages, and a de-referencing context for Reference elements.

MessageReference processing semantics are unambiguous: it must be possible for a message processor to dereference the contained Reference elements from the context of any of the messages identified by the MessageReference. A MessageReference must have runId and messagenumber attributes that identify a de-referencing context for the immediate recipient. The runId and messageNumber may identify the current message or another previously received message or some future message. A MessageReference may also contain a MessageList element that specifies the numbers of all the messages in the identified protocol run that can be used as de-referencing contexts for the contained Reference elements. An important feature of the MessageReference element is that it is protocol independent. Given a message identified by the MessageReference it is possible to process the enclosed Reference elements according to core XML Signature processing semantics. We can also express complex protocol tokens with different security properties by exploiting the indirect referencing provided by XML Signature and at the same time preserving our unambiguous processing semantics.

Figure 20 is an extract of an instance of the third message of the Coffey-Saidha protocol from Figure 7. In this message, the sender (the TTP) provides a digest of the payload (the SOAP message body) for the recipient to sign. The digest is included in a ReceiptsRequired element that uses a MessageReference element to declare that the payload will be available to the recipient in message 6 . The Messagelist element indicates that the payload is available in both protocol messages 1 and 6 . The URI in the enclosed Reference element refers the recipient to the "\#payload" fragment of message 6 . Note that the body of message 3 is empty. 


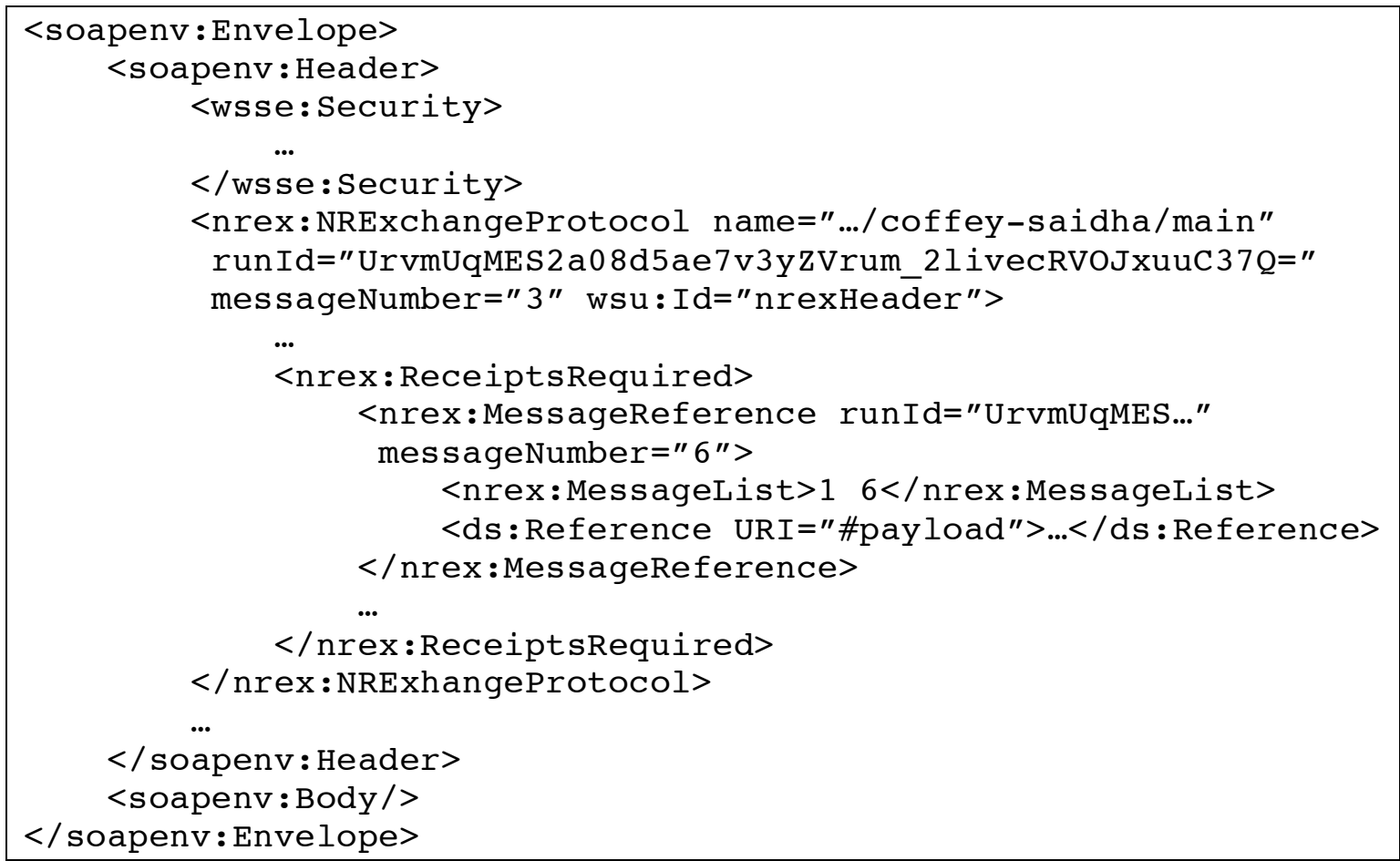

Figure 20: Example message 3 of with forward reference

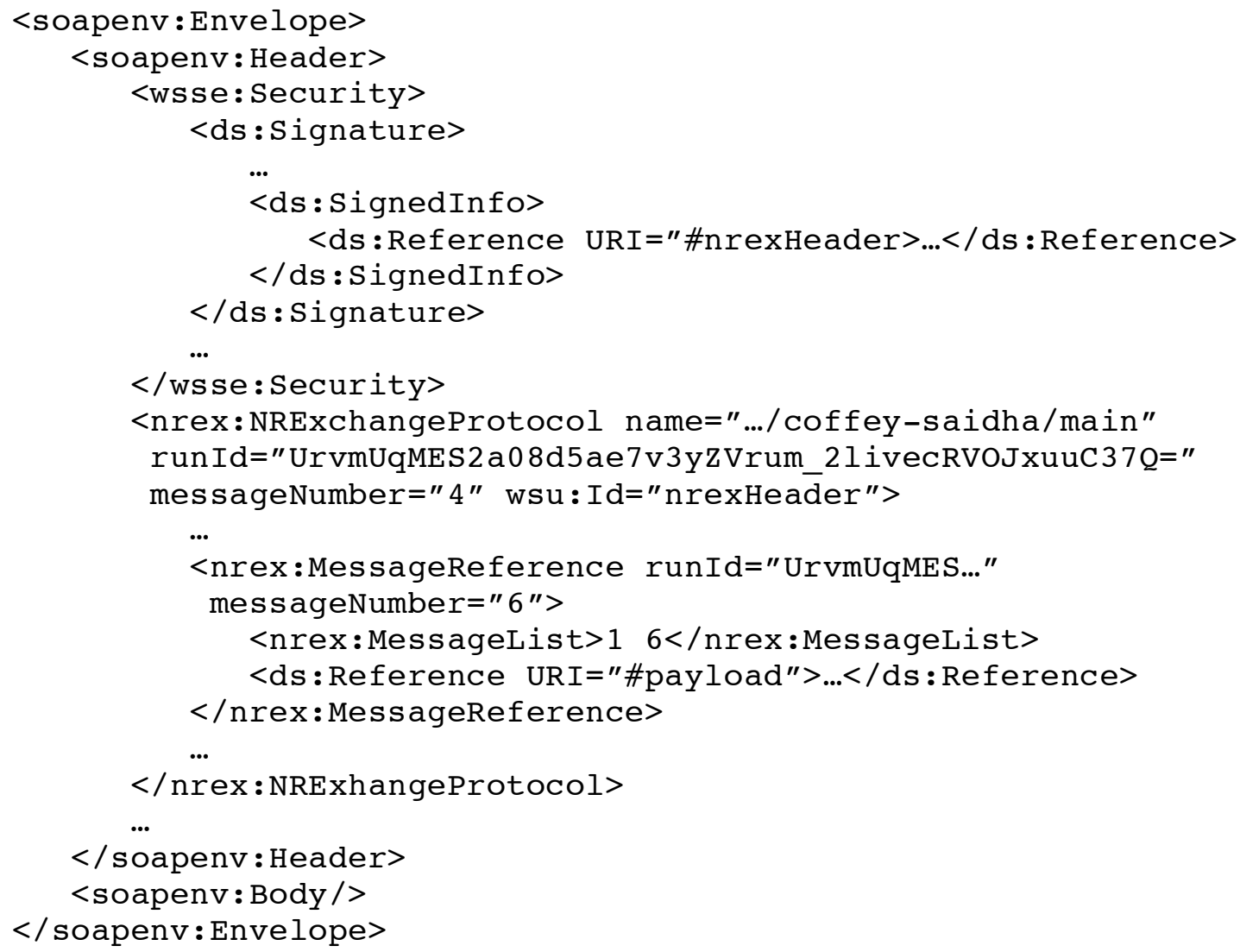

Figure 21: Example message 4 to receipt Messagereference and payload

As shown in Figure 21, the recipient includes the MessageReference element from message 3 in the NRExchangeProtocol header of their response. The whole header is signed. The SignedInfo element contains a Reference element that identifies the "\#nrexHeader" fragment as input to the signature. Since the MessageReference element 
contains a digest of the payload that will be provided in message 6 , the signature over the NRExchangeProtocol header represents the receipt requested in message 3 . The TTP relays this information to the original sender in message 5. The inclusion of the MessageList in the MessageReference allows the original sender to verify the receipt with respect to message 1 of the protocol run. Finally, the TTP sends the payload to the intended recipient in message 6 . Note the non-empty body of message 6 in Figure 22.

The use of the MessageReference element in the preceding examples allows the TTP to explicitly indicate that the recipient is to provide a receipt for the SOAP body of message 6 . The element also provides the recipient with the necessary input (h(payload)) for the signature and to construct the receipt without requiring access to the payload. The inclusion of MessageList indicates to other parties that they can find the same content in any of the other messages in the list to which they have access. In fact, the MessageList is an assertion that the identified input to message digests is identical whichever message in the list is used as a de-referencing context.

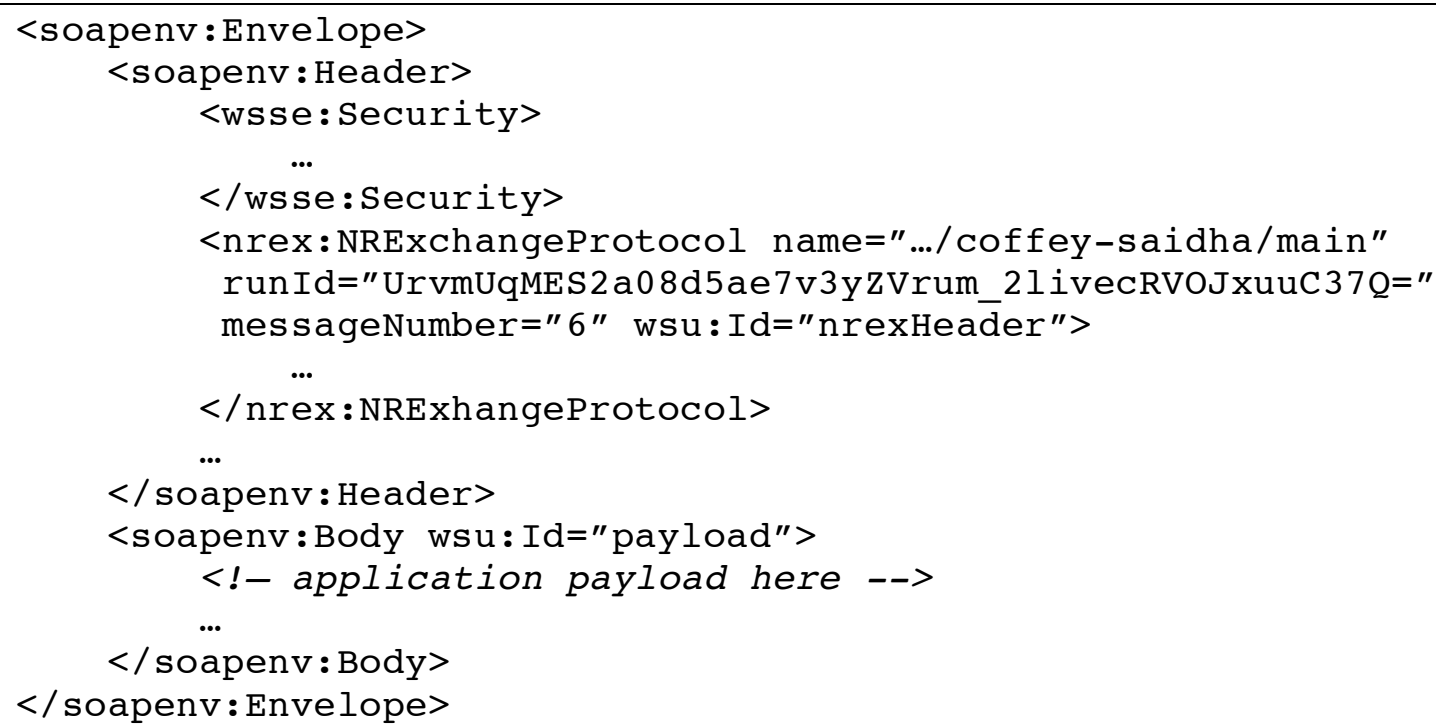

Figure 22: Example message 6 to provide payload

\section{Protocol message handling}

We now describe the processing of an incoming protocol message to illustrate the adaptability of the middleware to different protocols.

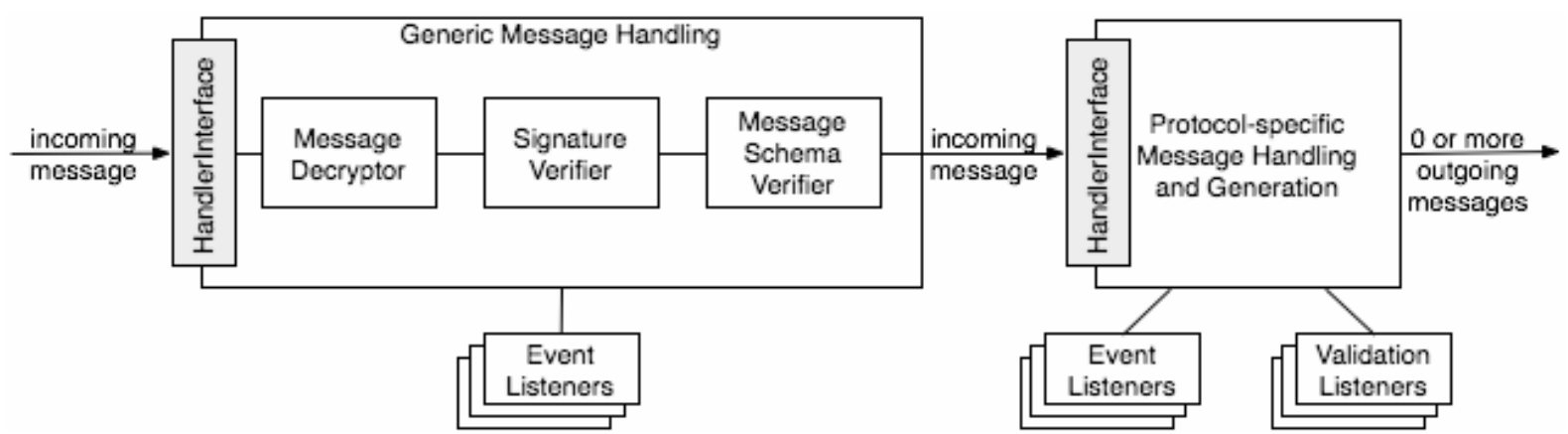

Figure 23: Message processing overview

As shown in Figure 23, there are two phases to message processing: generic message handling and protocol-specific message handling. Both phases provide the same handler interface to receive incoming messages for processing. In addition, event listeners can be registered in each phase to customise reaction to protocol progress. 
In the generic message handling phase, an incoming message is subject to processing that is common to all protocols. First, given access to appropriate keys, any encrypted elements of a message that it is possible to decrypt are decrypted. Then, any signatures contained in the message are verified, along with verification of associated time-stamps and certificates. These first two steps are essentially concerned with processing information in the WSSecurity header of a message. Assuming this succeeds, the message is validated against the NRExchange message schema. If any step of generic message handling fails then message processing terminates. This early termination of message processing may indicate an attempt to cheat and, therefore, may trigger dispute resolution (possibly including a request to abort an exchange). One of the uses of event listeners is to allow dispute handling to be triggered at the application-level in response to processing failures. If generic handling completes successfully, the incoming message is passed to a protocol-specific handler. The protocol name obtained from the header of the incoming message determines which protocol-specific handler is instantiated.

In the protocol-specific handling phase, the handler first determines the protocol run to which the messages relates and accesses the message log to determine the state of the protocol run. Since protocol messages are self-describing, the state of a protocol run is completely described by the set of messages that have been logged for that run. Given the state of a protocol run, the handler can determine whether the incoming message is in correct sequence. If the message is in correct sequence, the handler can: (i) verify that all expected protocolspecific tokens (message digests, random numbers etc.) are present; and (ii) verify the integrity of the tokens and, in particular, perform any necessary comparisons with tokens from any earlier messages. Assuming the message is valid with respect to the executing protocol, the protocol handler generates and sends any new protocol message(s) that are required to continue protocol execution. If the message is not valid with respect to the executing protocol, then, as for generic handling, processing terminates and dispute resolution may be triggered. As shown in Figure 23, at the appropriate point in protocol execution, the protocol-specific handler invokes application-level message validation via any registered validation listeners.

Both the GenericHandler and the ProtocolHandler provide an interface corresponding to the abort and resolve operations defined in Figure 17. Again, generic processing of a message is followed by instantiation of an appropriate protocol-specific handler from the protocol name obtained from the incoming message.

\section{Validation listeners}

A key requirement is for the middleware to be able to trigger run-time validation of business messages with respect to application-specific constraints (or rules) and, if performed, that this validation is non-repudiable. As shown in Figure 23, validation is therefore an integral part of message processing and of execution of the protocol chosen to implement run-time monitoring and enforcement. However, if validation logic is application-specific it cannot be fixed for a given protocol and hard-coded as part of the protocol implementation. To address the problem of integrating validation with protocol execution, the middleware supports the configuration of protocol handlers to instantiate one or more validation listeners that are specific to the given business interaction. These listeners are invoked at the appropriate point in protocol execution to obtain a validation decision that can then be incorporated as the payload of a validation message. The application programmer, and not the protocol implementer, is responsible for the implementation and the identification of the validation listeners that the protocol handler should instantiate. 


\section{Summary}

In this section, we presented our flexible, standards-based middleware for non-repudiable interactions between and with Web services. The middleware addresses the requirements identified earlier by allowing the "plugging-in" of non-repudiation protocol implementations to suit the given application context. The middleware also provides a general and systematic mechanism for the validation of business message exchanges with respect to applicationspecific constraints.

\section{CONCLUSIONS}

In this chapter, we have developed an approach and a reference implementation for the support of non-repudiable B2B interactions. We identified the need for different underlying mechanisms to suit different relationships between participants in a B2B interaction and the need to be able to adapt to the different capabilities of business partners. We described how existing protocols meet some of the identified requirements and presented modifications of the protocols to address others such as incorporation of application-level validation and adaptation for lightweight participants. The description of our Web services based implementation provided a general overview of the architecture showing how our services interact with existing Web service standards and services. We addressed the limitations of the XML Signature standards with respect to fair non-repudiation protocols. We described our Web services interface to protocol execution, the protocol message schema and the message handling process. We are not aware of another middleware system that is capable of rendering arbitrary Web service interactions both non-repudiable and fair. Further, we know of no other system that provides the flexibility to "plug-in" both the application-level validation of $\mathrm{B} 2 \mathrm{~B}$ interactions and the mechanism that enforces that validation at run-time.

Our implementation operates a layer above reliable messaging. However, there is duplication of effort between the non-repudiation protocols and reliable messaging both in terms of acknowledgements generated and message persistence. Further work could investigate tighter integration of the two services. The approach should be modular and configurable. Either the fair exchange service can provide reliable messaging directly (for greater performance) or, as now, it will rely on an existing standards-based implementation to provide a reliable channel.

The correct implementation of non-repudiation protocols is a challenging task. To gain high confidence in a given implementation, ideally we require both: (i) a formal, verified protocol specification, and (ii) tool support to generate an implementation from the specification. The former area has attracted considerable attention. Thus, another area for future work is tool support for the generation of protocol implementations for execution in our framework. We need to investigate the automation of as much of the implementation process as possible and to provide guidance on those aspects that cannot be automated.

\section{REFERENCES}

Apache Software Foundation. (2005a). AXIS Web Services. http://ws.apache.org/axis/. Apache Software Foundation. (2005b). Tomcat Servlet Container. http://tomcat.apache.org/. Asokan, N. (1998). Fairness in Electronic Commerce (Research Report RZ3027): IBM Zurich Research Lab.

BEA. (2005). WebLogic 8.1 Trading Partner Integration. http://edocs.bea.com/wli/docs $81 /$ pdf/tpintro.pdf. 
Bilorusets, R. (2005). Web Services Reliable Messaging Protocol (WS-ReliableMessaging) (Specification). http://www-128.ibm.com/developerworks/library/specification/ws-rm/: BEA, IBM, Microsoft and TIBCO.

Christensen, E., Curbera, F., Meredith, G., \& Weerawarana, S. (2001). Web Services Description Language (WSDL) 1.1 (W3C Note). http://www.w3.org/TR/wsdl.

Coffey, T., \& Saidha, P. (1996). Non-repudiation with mandatory proof of receipt. $A C M$ SIGCOMM Computer Communication Review, 26(1), 6-17.

Cook, N., \& Robinson, P. (2006). NRExchange: XML schema and WSDL for Web service non-repudiation protocols. http://homepages.cs.ncl.ac.uk/nick.cook/ws-nrex/.

Cook, N., Robinson, P., \& Shrivastava, S. K. (2006). Design and Implementation of Web Services Middleware to Support Fair Non-repudiable Interactions. International Journal of Cooperative Information Systems (IJCIS) Special Issue on Enterprise Distributed Computing, 15(4), 565-597.

DataPower. (2004). XML Security Gateway. http://www.datapower.com/products/xs40.html. Eastlake, D., Reagle, J., Imamura, T., Dillaway, B., \& Simon, E. (2002). XML Encryption Syntax and Processing (W3C Recommendation). http://www.w3.org/TR/xmlenc-core/: W3C. Eastlake, D., Reagle, J., \& Solo, D. (2002). XML-Signature Syntax and Processing (IETF RFC 3275 and W3C Recommendation). http://www.w3.org/TR/xmldsig-core/: IETF/W3C. Even, S., \& Yacobi, Y. (1980). Relations Among Public Key Signature Systems (Technical Report CS175). Haifa, Israel: Technion Israel Institute of Technology.

Ezhilchelvan, P. D., \& Shrivastava, S. K. (2005). A Family of Trusted Third Party based Fair-Exchange Protocols. IEEE Transactions on Dependable and Secure Computing, 2(4), 273-286.

Fischer, M. J., Lynch, N. A., \& Paterson, M. S. (1985). Impossibility of Distributed Consensus with One Faulty Process. Journal of the ACM, 32(2), 374-382.

Grangard, A., Eisenberg, B., \& Nickull, D. (2001). ebXML Technical Architecture Specification v1.0.4 (OASIS Final Draft). http://www.ebxml.org/.

Gravengaard, E., Goodale, G., Hanson, M., Roddy, B., \& Walkowski, D. (2003). Web Services Security: Non-Repudiation (Proposal Draft). http://schemas.reactivity.com/2003/04/web-services-non-repudiation-05.pdf.

Gudgin, M., Hadley, M., \& Rogers, T. (2006). Web Services Addressing (WS-Addressing) (W3C Submission). http://www.w3.org/TR/ws-addr-core/.

Hallam-Baker, P., \& Mysore, S. (2005). XML Key Management Specification (XKMS 2.0) (W3C Recommendation). http://www.w3.org/TR/xkms2/.

Housley, R., Ford, W., Polk, T., \& Solo, D. (1999). Internet X.509 Public Key Infrastructure: Certificate and CRL Profile (IETF RFC 2459): Internet Engineering Task Force.

Josefsson, S. (2003). The Base16, Base32, and Base64 Data Encodings (IETF RFC 3548): Internet Engineering Task Force.

Kremer, S., Markowitch, O., \& Zhou, J. (2002). An Intensive Survey of Fair Non-repudiation Protocols. Computer Communications, 25(17), 1601-1621.

Markowitch, O., Gollmann, D., \& Kremer, S. (2002). On Fairness in Exchange Protocols. In Proceedings of 5th International Conference on Information Security and Cryptology (ISISC 2002), Seoul, Korea.

Markowitch, O., \& Roggeman, Y. (1999). Probabilistic Non-repudiation without Trusted Third Party. In Proceedings of 2nd Workshop on Security in Communication Networks, Amalfi, Italy. 
Milosevic, Z., Gibson, S., Linnington, P. F., Cole, J., \& Kulkarni, S. (2004). On design and implementation of a contract monitoring facility. In Proceedings of 1st IEEE Workshop on Electronic Contracting, San Diego, CA, USA.

Minsky, N., \& Ungureanu, V. (2000). Law-Governed Interaction: A Coordination and Control Mechanism for Heterogeneous Distributed Systems. ACM Transactions on Software Engineering and Methodology, 9(3), 273-305.

Molina-Jimenez, C., Shrivastava, S. K., \& Warne, J. (2005). A Method for Specifying Contract Mediated Interactions. In Proceedings of 9th IEEE International EDOC Enterprise Computing Conference, Enschede, Netherlands.

Nadalin, A., Kaler, C., Hallam-Baker, P., \& Monzillo, R. (2004). Web Services Security: SOAP Message Security 1.0 (OASIS Standard). http://docs.oasisopen.org/wss/2004/01/oasis-200401-wss-soap-message-security-1.0.pdf.

Nenadic, A., Zhang, N., \& Barton, S. (2004). FIDES - A Middleware E-Commerce Security Solution. In Proceedings of 3rd European Conference on Information Warfare and Security (ECIW), London, UK.

Pagnia, H., \& Gärtner, F. (1999). On the impossibility of fair exchange without a trusted third party (Technical Report TUD-BS-1999-02): Department of Computer Science, TU Darmstadt.

Periorellis, P., Cook, N., Hiden, H. G., Conlin, A., Hamilton, M. D., Wu, J., et al. (2006). GOLD Infrastructure for Virtual Organisations. In Proceedings of 5th UK e-Science All Hands Meeting, Nottingham, UK.

Perrin, T., Andivahis, D., Cruellas, J. C., Hirsch, F., Kasselman, P., Kuehne, A., et al. (2004). Digital Signature Service Core Protocols, Elements and Bindings (OASIS Committee Working Draft). http://www.oasis-open.org/committees/dss.

RosettaNet. (2005). eBusiness Standards for the Global Supply Chain. Retrieved 1

December, 2005, from http://www.rosettanet.org/RosettaNet/

Schneier, B. (1996). Applied Cryptography: John Wiley and Sons.

Verisign. (2004). Verisign Trust Gateway: Simplifying Application and Web Services Security (Verisign White Paper). http://www.verisign.com/products-services/securityservices/intelligence-and-control-services/application-security/index.html.

Wang, G. (2005). Generic Fair Non-repudiation Protocols with Transparent Off-line TTP. In Proceedings of 4th International Workshop for Applied PKI, Singapore.

Zhou, J., \& Gollmann, D. (1997). Evidence and non-repudiation. Journal of Network and Computer Applications, 20(3), 267-281. 